\title{
"ITT: Rape Analysts" \\ Hosting Negotiations of Consent, Kink, and \\ Violence in Virtual Space
}

by

\section{Lauren Menzie}

A thesis submitted to the Faculty of Graduate and Postdoctoral Affairs in partial fulfillment of the requirements for the degree of

Master of Arts

in

Law and Legal Studies

Carleton University

Ottawa, Ontario

(C) Lauren Menzie 2018 


\begin{abstract}
This project examines the use of virtual space in navigating the meaning of consent after the fact. Recently, some individuals have begun to interrogate their own past sexual experiences through the use of online sex education forums, blogs, and subreddits. Through this process of eliciting community feedback, many have (re)framed these experiences as consensual or nonconsensual. Using data that was obtained from five distinct Reddit communities (or subreddits), comprised of 66 posts and over 4,000 comments, this thesis examines this process as both an extralegal, reflexive negotiation of consent, and a user-constructed virtual trial. This discussion posits that the use of these spaces (rather than traditional legal/institutional resolutions) is a response to the significant changes in how consent has been conceptualized within the mainstream; this is exemplified through academic literature theorizing consent and the shifts in Canadian governance of sexual assault.
\end{abstract}




\section{Acknowledgements}

This work would not have been possible without my supervisor and friend, Professor Ummni Khan. Ummni, thank you for putting up with my far-reaching (and perpetually extending) deadlines, and for the encouragement, insights, and guidance you've provided. Thank you to my committee, Professor Rena Bivens and Professor Jena McGill, for your time, your advice, and for the support and challenges you've brought to this project.

I am immensely grateful for all my teachers, professors, and mentors who have supported me over the years. Richard, I cannot express what a huge source of support you have been, nor the influence you have had on my work. Dale, I have you to thank for insights in qualitative methods, and for your friendship and encouragement. I would also like to thank the larger academic communities I am privileged to be a part of; thank you to the faculty of Criminal Justice at The University of Winnipeg, as well as the Law and Legal Studies faculty at Carleton University.

Thank you to my cohort for contributing to a stimulating academic environment. To Darby, Deepa, Taryn, and the many that I've inevitably missed, thank you for your influence on my work and, more importantly, for the much-needed breaks from my work.

Alex, my soul sister, you are everything to me. Our Southdale Tim's dates turned Skype dates have always been the highlight of my week. You were the first person to read this project, and your support has meant the world. Nicole, my odd bird, your compassion for the people in your life is inspiring. The carefully worded critiques and perspectives that you have brought to this work has undoubtedly benefited it. I treasure you both.

Thank you to my parents, Kim and Gord, for their support, encouragement, and love. My mother, particularly, for strengthening my intellectual curiosity at an early age by answering all my legal questions with "I can't tell you that, it's confidential." I'd also like to thank my grandparents - two of my absolute favourite people - for their love, their support, and for taking care of my cacti. Duncan, thank you for the help with online terminology but, more importantly, thank you for being the best brother and friend anyone could ask for. I love you all.

To my partner, Nolan, thank you for being there. Thank you for helping with my legal citations, for patiently listening whenever I wanted to talk through my work, and for all the times we've gone for oysters. Thank you for always being my loudest voice of support. I love you.

Andy and Karrol, thank you for your love, support, and for making me feel like family.

And, of course, thank you to all the dogs in my life. To Isla, Rusty, Bear, and Thor, thanks for the enthusiasm, love, and for never asking me about my thesis. Most of all, I have to thank my best girl, my Moka, for giving me unconditional love, immeasurable support, company on long walks, and an occasional cuddle. I am forever grateful. 


\section{Table of Contents}

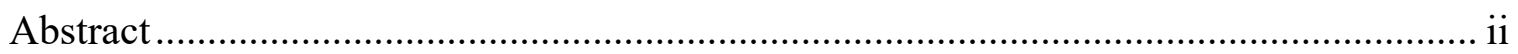

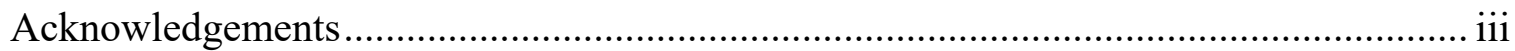

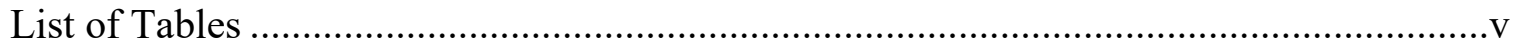

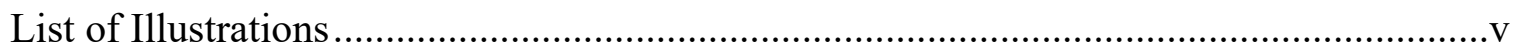

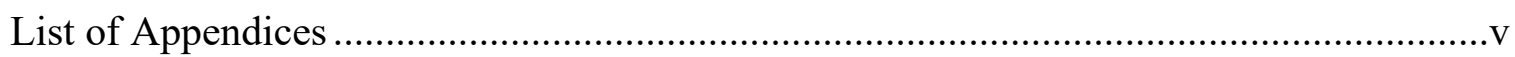

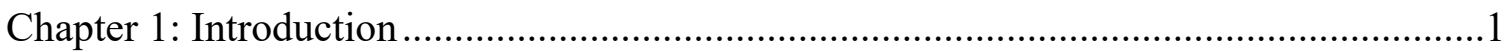

Chapter 2: Literature Review and Theoretical Framework ...............................................10

From Rape to Sexual Assault: Codified Law.............................................................. 10

Sexual Assault in the Common Law ................................................................... 12

Theoretical Section: Harm, Judging, and Belonging ................................................ 21

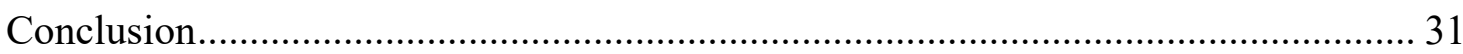

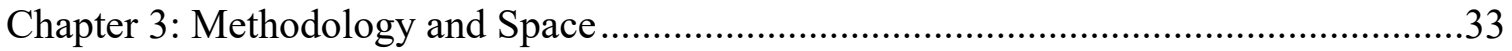

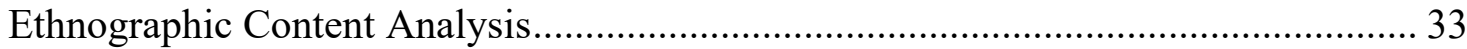

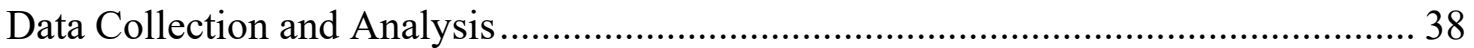

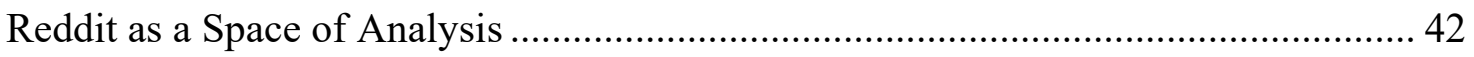

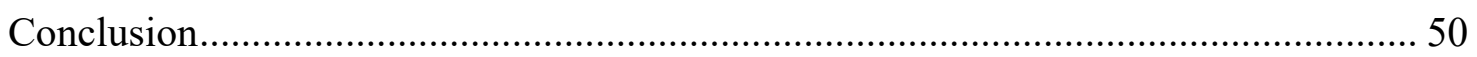

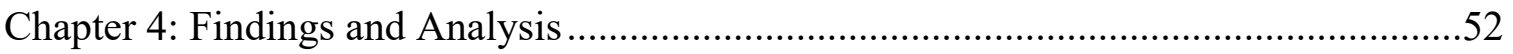

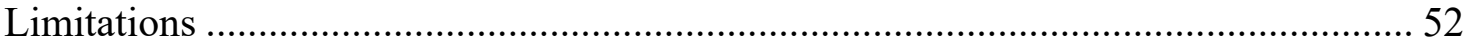

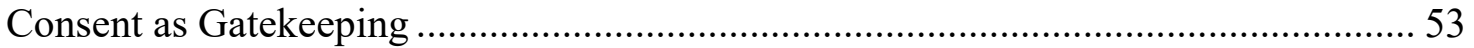

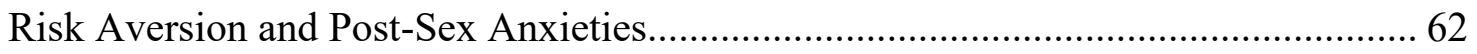

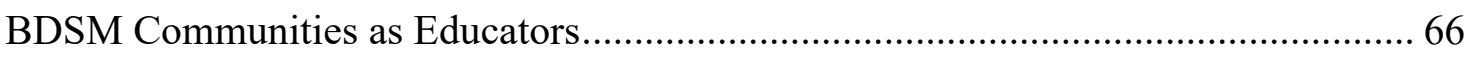

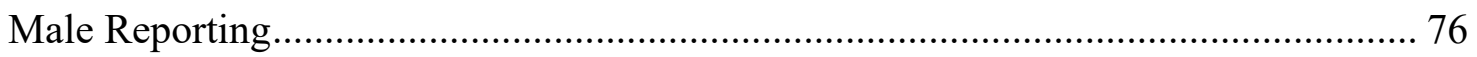

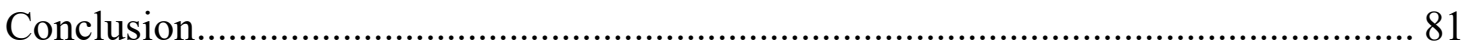

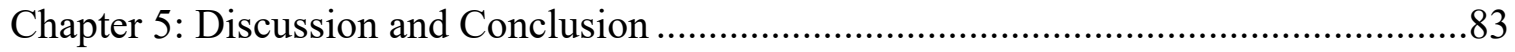

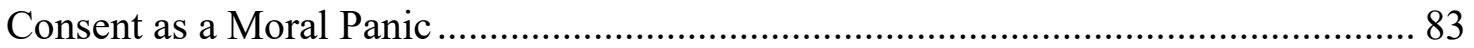

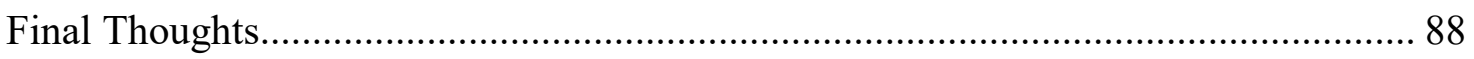

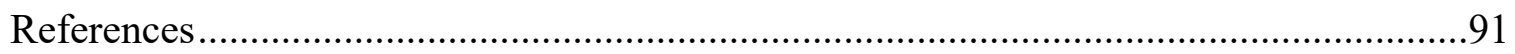

Legal Cases and Factums ........................................................................................ 91

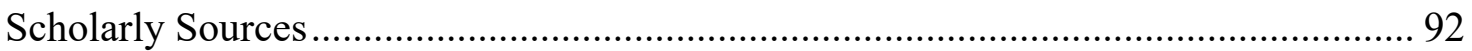

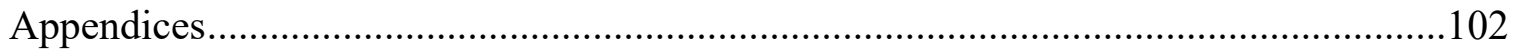

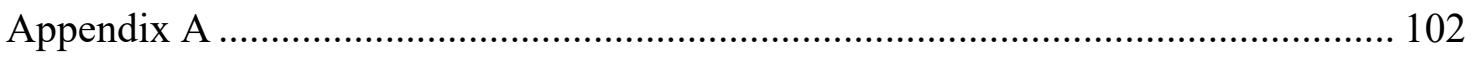




\section{List of Tables}

Table 1: Representation of OPs by Gender and Role (Victim or Perpetrator)............77

\section{List of Illustrations}

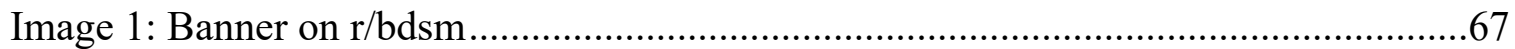

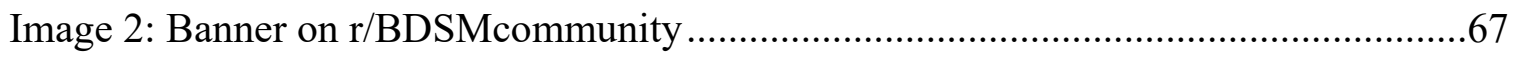

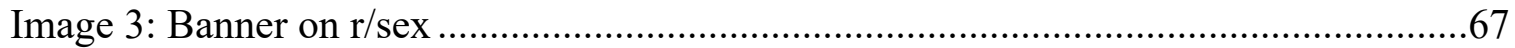

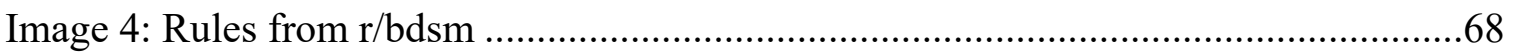

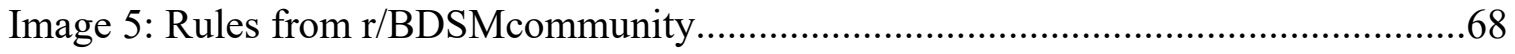

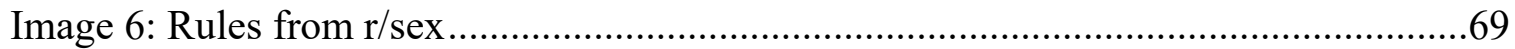

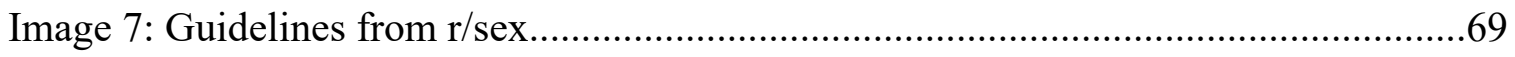

\section{List of Appendices}

Appendix A - Coding Index................................................ 102 


\section{Chapter 1: Introduction}

My title begins with a quote, "ITT: Rape Analysts." ITT, an acronym meaning "in this thread," speaks to the nature of online spaces and the conversations that emerge from discussion forums, whereas "rape analysts" would be persons predominantly concerned with assessing sexual harms. This thesis is concerned both with the nature of virtual spaces and the conversations that emerge from them. It interrogates the use of online communities to negotiate and navigate questions of sexual violence, consent, kink, and harm. It further examines the process of retroactively deciding whether a sexual experience was consensual and how sexual scripts can be rewritten and reframed with the help of an online community. "ITT: Rape Analysts" was one comment that emerged from my extensive data collection conducted on Reddit. My concern was with the visible emergence and use of these spaces to question the nature of personal sexual experiences to a broader community of virtual jurists. This thesis looks to these communities and how they balance social and legal understandings of consent and sexual violence along with the interests and needs of the original poster.

Online spaces such as subreddits - as well as blogs, sexual education forums, and advice columns - are now virtual spaces where individuals may interrogate their own sexual experiences and (re)frame them as either consensual or nonconsensual. This process, despite being unconventional and perhaps unfamiliar within a traditional justice-seeking praxis, in many ways mirrors conventions of a criminal trial. There is a formalistic system of rules that govern the space, the presentation of a fact scenario, and the 
opportunity for community participation - not unlike a jury trial. Reddit facilitates space for distinct subcommunities to create their own rules and customs for its users in engaging with these posts, resulting in data that follows a clear and expected structure. Within these subcommunities, users are able to disseminate and engage with pre-existing content and posts. However they can also use these forums as platforms to create and share their own content, educate other users, provide advice, and ask questions. It is this communicative process, and the specific questions asked and addressed, that is the focus of this thesis.

This thesis, as exemplified through the title, uses language that may be unfamiliar to readers who are not practiced Reddit users. Reddit refers to itself as the front page of the internet (Massanari 2015), but can be more technically described as a link aggregation website with a comments section. On Reddit, users are commonly referred to as "Redditors." Accordingly, I use the term Redditor to refer to any user who has commented on a post. Creators of posts are referred to as "original posters" or more often by the abbreviation "OP." While OPs are also Redditors, I distinguish between the two in my thesis as they have unique roles in the discussion. Users can surf Reddit without an account, but many users will choose to have accounts so that they can subscribe to content of interest. Reddit hosts many wide-ranging subcommunities, referred to as "subreddits," which users can subscribe to in order to curate their front page with content of interest. Reddit also uses a voting algorithm to hierarchically organize content. Votes are measured by "karma points," and users are able to "upvote" or "downvote" content 
and comments to show their level of interest and approval. Reddit then uses this information in how it presents and orders content.

Interest and critical attention to issues of sexual violence has flared up at various points in time. ${ }^{1}$ Issues surrounding consent are now topical discussion points, driven predominantly by the recent \#MeToo movement. Public disclosures of sexual violence, or the "outing" of celebrities and public figures as rapists or perpetrators of sexual violence has been receiving widespread media attention. This attention has prompted many to question what constitutes the difference between violent or "bad" sex. Prior to this, the interactions between sexual violence, consent, and law have received wideranging, diverse coverage in academic work. It has been said that the defining characteristic of sexual violence is the absence of consent (Beres 2007). This understanding prompts a consideration of spontaneous sociology; we have adopted a common sense meaning and framework without critically reflecting on the cultural, historical and social contexts from which these understandings arose (Bourdieu 1991; Beres 2007, p. 95).

In fact, sexual consent itself has stimulated a great deal of debate and public interest throughout the last decade (Cowling and Reynolds 2016). Frameworks for sexual consent have been advanced and critiqued amongst interdisciplinary audiences, many noting a

\footnotetext{
${ }^{1}$ For instance, Katie Roiphe documents the rising attention to campus sexual violence and date rape in her book The Morning After: Sex, Fear, and Feminism (1994). Roiphe critiqued the social perception and representation of campus sexual violence and the then current cultural preoccupation with victimization. She further argued that this echoed a great deal of outdated assumptions surrounding how men and women experience sex, positioned women as fearful of their sexuality, and placed a heightened interest on sexual regulation.
} 
gendered dynamic to consent and inherent power structures. Some dominance feminists have questioned the ability of women to give consent under patriarchal structures entirely (MacKinnon 1989). Demystifying and understanding sexual consent decisions and how they are made brings with it the possibilities of enhancing ethical conduct and uncovering important dynamics of our interpersonal and romantic lives (Cowling and Reynolds 2016). Sexual consent is often thought of as either present or absent, and, following this logic, sex is wanted or unwanted. This conceptualization has received very little challenge, however some limited scholarship has pointed towards a missing discourse of ambivalence.

Muehlenhard and Peterson (2005) attempted to trouble the dominant conceptualization of sex as either wanted or unwanted (consensual or nonconsensual) by pointing to a body of research that demonstrates feelings of ambivalence concerning sex. The discourse of ambivalence came out of prior research that explored tensions and confusion in wanting (and not wanting) sex. Specifically, the research has explored the prevalence and experience of "token resistance," a term used to describe the situation when the individual desires sex, but for a variety of reasons, says "no" to sexual advances. Rather than working to remedy the dichotomous nature of both wanting and unwanting, Meuhlenhard and Peterson (2005) fragmented the wanting and unwanting by considering the potential outcomes and implications of sexual contact. Here, ambivalence is theorized to emerge from the consequences of sex, and inquiries into the nature of consent are again based upon a fairly limited framework. Instead of a dichotomous analysis, there are now four ways to understand sexual contact: as wanted and consensual; as wanted and 
nonconsensual; as unwanted and consensual; as unwanted and nonconsensual. While this analysis attempts to nuance our understanding of ambivalence due to potential outcomes that may trouble wanting or not wanting sex, it still relies upon discrete categories of experience and removes the temporality and flexibility of consent and wanting as it manifests in an interaction. Sex is seen as a relatively static performance, where the status of consent and wanting remains a constant throughout. Law, despite being frequently critiqued for lacking subjectivity in understanding consent, acknowledges that sexual consent is a dynamic process. Here, however, law stresses the importance of ongoing, active consent, which is further troubled by Meuhlenhard and Peterson's (2005) findings that consent may be present when sex is unwanted, or absent when sex is wanted.

This project seeks to contribute to the work that muddies the dichotomous nature of consent by examining the way online users turn to Reddit as an epistemic tool to make sense of sexual experiences. To date, I have been unable to find any academic treatment of these discussions and how online users negotiate and label their experiences with guidance and judgement from a community. This thesis examines the use of online spaces to question sexual consent after the fact, and casts these conversations as virtual trials. It looks at discussions surrounding sexually ambiguous or grey encounters and how users solicit advice from broader communities well practiced in judging. Both the advice and the virtual forum are considered forms of extralegal authority, which includes both "majority" perspectives as well as "dissenting" ones. The purpose of this thesis is to explain the current use of these spaces, their operation, and what can be gleaned about the conundrum of consent from studies in virtual communities and practices of judging. 
My interest in these spaces came about through my previous work exploring the legal governance and denunciation of sexuality, and imagining the law as a precautionary, riskaverse instrument. Initially, my project was concerned with the Canadian common law and its increasingly stringent conceptualization of consent in cases of sexual assault. In attempting to unpack the sociolegal and interpersonal challenges that would come from a widespread societal adoption of this framework, I was led to users who had similar concerns and questions online. For these reasons, and despite the fact that online extralegal jurisprudence has no clear jurisdiction, I have chosen to situate this work within a Canadian legal framework. The focus here is not to advocate for a true articulation or construction of consent, but rather to unsettle the current legal framework and explore an alternative means to navigate questions of consent.

The second chapter of this thesis seeks to explain the sociolegal and theoretical context in which these discussions emerge. It begins by tracking the common law's engagement and (re)interpretation of consent within Canadian law and outlines the current legislative framework for sexual assault. Canadian law is argued to have taken a precautionary approach, requiring affirmative consent that cannot be implied or given in advance. These consent restrictions are also extended to those who grant consent, which may lead to doubting one's agency and capability to consent to sex. A sexual assault charge will challenge an alleged perpetrator's sense of belonging and citizenship as they are subjected to denunciation and legal governance. This thesis argues that there will be similar challenges to the identity of victims of sexual violence as rape has been 
understood in both law and society to fundamentally and permanently change a person. The use of Reddit to navigate questions of consent and sexual assault is seen as a means of maintaining or redefining citizenship, as well as self-understanding. Users enter these spaces attempting to gain clarity about a past event in communities that are well practiced in judging.

In the third chapter, I outline my methodological approach to analyzing discussions on Reddit. This project was accomplished through an ethnographic content analysis (ECA) of virtual trials taken from five distinct subreddits, which included 66 posts and over 4,000 comments. In this chapter, I also discuss my perspective on Reddit as a space of analysis and challenge popular academic imaginings of Reddit and its users. Through my exposure and immersion in these communities, Reddit users demonstrated themselves to be complex and dynamic, particularly in the subreddits where I collected my data. I justify my inclusion criteria for data and how subreddits were selected for analysis.

The fourth chapter presents the findings derived from analysis. The analysis concerns itself both with how users present and respond to fact scenarios. Common themes provided insights into how users understood consent and the sexual scripts that governed giving and reading consent. Users who posted and commented in these spaces also demonstrated risk-averse perspectives when granting and reading consent or having sex, and significant anxiety after, what has been termed, "grey" sexual encounters. In these circumstances, users emphasized their need to use a label (i.e. rape) to define their experiences in order to make sense of otherwise ambiguous or sexually uncomfortable 
encounters. While many communities addressed questions through surface level answers, users on BDSM affiliated subreddits chose to disseminate educational information and challenge pre-existing consent frameworks. Although they would uphold the legitimacy of acts that are criminal in a Canadian legal framework, they did so while advancing a "safe, sane, and consensual" model of consent. They also were quick to condemn and exclude members of their community who were accused of sexual violence; this, along with the consent frameworks advanced, suggest that these communities mobilize similar logics to that of the mainstream. This may be a means of integrating or seeking inclusion and acceptance. Similarly, men found a much more receptive audience when reporting or questioning sexual assaults on Reddit. Past research has demonstrated that men face unique and pervasive challenges when reporting sexual assaults. By curating and hosting a virtual trial, men may find a more sympathetic and receptive audience.

Chapter five is a concluding chapter which includes an extensive discussion and argument that consent culture today may be building towards a moral panic. Many current headlines attempt to widen the reach of sexual consent and further entrench systems of governance through the use of consent apps and "consent captains". While there is an important social value in respecting the consent of others, the current social preoccupation with these questions demonstrates heightened risk-aversion, nuancedestroying and anxiety-provoking attitudes towards sex.

It could be asked why this project invests in considering discussions on Reddit as a jurisprudential representation or trial. Some of these posts have, and still may, reach 
substantial audiences, and may play a role in how readers go forward and construct their own narratives about their experiences. During data collection, it became evident that these conversations occur in many diverse, wide-ranging subreddits. ${ }^{2}$ The subreddits that had been examined had just over three million combined subscribed members. ${ }^{3}$ These posts are overwhelmingly influential in scope; they also may be overwhelmingly influential for the OP who chooses to host a virtual trial. The comments provided often give the OP license to label or reframe their experience, and some OPs later commented that feedback led them to therapy or encouraged them to report the assault. These communities provide clarity to the $\mathrm{OP}$ as well as to broader community members as to how consent and sexual assault should be understood and articulated in legal, moral, and philosophical contexts. These discussions may serve as an introduction to complicated jurisprudential issues, frameworks, and value systems. Finally, the use of these spaces rather than traditional means of justice is significant as it is a subtle rejection of that system as one of sole authority or value to the OP.

\footnotetext{
${ }^{2}$ The majority of these subreddits were not examined as they fell outside of the data selection criteria. This will be discussed further in Chapter 3.

${ }^{3}$ As of June 23, 2018, r/sex had 940,392 subscribed members, r/relationships had 1,290,130 subscribed members, r/relationship advice had 623,754 subscribed members, r/bdsm had 125,825 subscribed members, and r/BDSMcommunity had 94,008 subscribed members. These posts may appear on any of these subscribed members 'front page' when they visit Reddit, and may further extend to other readers who lurk in these spaces.
} 


\section{Chapter 2: Literature Review and Theoretical Framework}

Canadian governance of sexual assault and offending has experienced dramatic changes over the past few decades. These changes, both codified and through common law interpretation, have fundamentally altered what constitutes sexual offending and what behaviours may be captured under the purview of criminal law. The discussion in this chapter intends to cover those changes, stressing the legislative and societal shifts in how we conceptualize consent, as well as laying the theoretical foundation of this thesis.

\section{From Rape to Sexual Assault: Codified Law}

Legislation governing sexual assault has a long, dynamic history in Canada. Canadian laws originally derived from the English common law where, in its nascency, sexual assault (then, rape), was thought to be a consequence from abduction (Tang 1998, p. 259). In the late thirteenth century, English lawmakers adapted the definition of raptus (ravishment) to conflate rape and abduction; this allowed for rape, coerced marriage, and consensual elopement to be captured by the statutes (Dunn 2007). Men were at the core of these legislative responses as they were the offended party through the loss and possible violation of their wives and property (Estrich 1987; Matlock 2014). When Canadian criminal law was developed, it thus carried with it this underlying patriarchal ideology, which entrenched problematic expectations of gender roles (Los 1994). The Criminal Code (C-127) was amended in 1983 intending to quash systemic rules that sustained bias against women and, in this process, rape was reclassified as a type of assault (Tang 1998). 
Governing sexual assault rather than rape prompted a more gender-neutral approach in law while simultaneously opening the scope of legislation to address a wider range of sexually assaultive behaviour. By gender-neutral, here the suggestion is that law began to pave the way for a more humane, just treatment of victims of sexual violence, recognizing that sexual violence may occur regardless of marital status or sexual history. In changing the language of the offense from rape to sexual assault, legislature shifted its consideration to the violence of the act rather than the sexual nature; here, the focus became more in tune with harm and the violation of sexual boundaries as a form of violence instead of focusing solely on penetrative sexual violence (Gunn and Minch 1998; Stuart 1992).

While rape prior to 1983 was a very recognizable offence, sexual assault offending today engages with a great deal of messiness, ambiguity, and grey areas of law. The understanding that women are not morally underdeveloped, are capable of providing credible testimony, and that reporting may not always be immediate following an assault when held against our legal standard of proving both mens rea in an accused and guilt beyond a reasonable doubt have resulted in a very nuanced and ever-evolving framework via the common law. A great deal of this confusion rests upon consent. Many legal scholars have argued that - at least on paper - recent common law decisions from sexual assault cases have resulted in an affirmative consent framework now held in Canadian law (Benedet 2014; Gotell 2008). Affirmative consent means that there is an active and voluntary communication of consent between both parties at each stage of sexual 
touching. This communication cannot be passive or assumed, and must be ongoing (see R. v. Ewanchuk 1999 and R. v. J.A. 2011). By imposing more specific criteria that must constitute consent, the common law furthered the scope of behaviours that may be subject to governance.

$\underline{\text { Sexual Assault in the Common Law }}$

There are several Supreme Court of Canada cases responsible for reinterpreting the common law iteration of consent and, on a more subtle level, how harm is understood by our courts. Canada's definition of sexual assault is quite broad, and captures all unwanted sexual activity. Here, the determining factor for what constitutes unwanted rests predominantly on consent, but also, on the legal impermissibility to consent under certain circumstances or to consent to harm.

For unwanted sexual activity to result in a criminal conviction, proof is required beyond a reasonable doubt of two basic elements: that the accused committed the actus reus (here, unwanted sexual touching); that the accused has the necessary mens rea. Mens rea to sexual assault cases would constitute the intention to touch while knowing, or being wilfully blind to, or reckless of, a lack of consent. Demonstrating that the actus reus has been committed requires the proof of three elements: touching; the sexual nature of the contact; the absence of consent. Both touching and the sexual nature of the contact were described by the court as objective standards, while the absence of consent relies on a determination of the complainant's subjective state of mind (R. v. Ewanchuk 1999). How 
the accused understood the complainant's state of mind is only relevant should a defence of honest but mistaken belief in consent be raised when assessing mens rea.

Ewanchuk is one of the leading cases that set forth how the courts must measure consent in cases where it is presented as a defence to sexual assault. At trial, the complainant was found to have provided the accused with "implied consent," a defence that had not and still does not exist within criminal law (R. v. Ewanchuk 1999, at para. 31). The Supreme Court in Ewanchuk found that this treatment focused on the potential to objectively construe consent on the part of the accused, and therefore erred in law by focusing the examination of consent on the behaviour of the complainant rather than her state of mind at the time. Put more simply, consent could not be inferred by an accused regardless of objectively present contextual clues. Consent must be established through the complainant's state of mind during the encounter; her behaviour is not relevant in establishing consent.

The Court stressed that the absence of consent, one of three elements to prove the actus reus of an offence, could solely be measured through the complainant's state of mind; therefore, the only direct source of evidence must come from their testimony. The Court also stressed that consent, in order to be legally effective, must be freely given $(R$. $v$. Ewanchuk 1999, at para. 36). In Ewanchuk, the complainant testified that, under the circumstances, she was afraid and therefore, throughout the sexual encounter, waited or chose not to voice her lack of consent. The Court found that this was one of many conditions where there were outside factors that may prompt apparent or visible consent 
and, as such, consent was not freely given (R. v. Ewanchuk 1999, at para. 36). Further, the Court ruled that the complainant's fear did not need to be founded or reasonable, nor did it need to be communicated to the accused in order to vitiate consent (R. v. Ewanchuk 1999, at para. 39).

The Court's finding that consent must be freely given is also contingent on them having an operating mind (R. v. J.A. 2011, at para. 36). An operating mind has yet to be qualified by the Court, however it has been made clear that, with respect to self-induced voluntary intoxication, being unconscious is not the only state capable of vitiating consent $(R$. $v$. Esau 1997). Intoxication that has been brought about involuntarily, for instance, drugs unknowingly administered by an accused, will always vitiate consent (R. v. Daigle 1997, aff'd 1998). However, Canadian law has struggled as of late to clearly articulate the relationship between a complainant's voluntary intoxication and consent (R. v. Al-Rawi 2018). This remains a contested and ill-defined point of law; section $273.1(2)(\mathrm{b})$ of the Criminal Code establishes that "no consent is obtained... where the complainant is incapable of consenting to the activity," however the Supreme Court has not authoritatively ruled on the dividing line between capacity and incapacity. An impairment of cognitive ability, short of loss of consciousness, is a grey area of the law in relation to a person's capacity to consent. Legal authorities have been unable to conclusively and unanimously decide this issue or introduce a test, however, legal intervenors have recently suggested that the test for incapacity should be:

(a) understanding of the nature of the specific sexual act in question; (b) ability to assess the risks and consequences associated with the act in the 
particular circumstances confronting the complainant, including physical risks (such as pregnancy or disease) and social risks (such as public exposure); (c) understanding that she has a choice as to whether to participate or decline to participate in the act; and

(d) capacity to communicate consent ${ }^{4}$ (R. v. Al-Rawi 2018, at para. 36)

The intervenors also suggested that the courts have historically overlooked the communicative nature of consent (R. v. Al-Rawi 2018, at para. 40). Although this framework in its entirety was ultimately rejected by the appellate court in $A l-R a w i$, the push to recognize communication as an essential element of consent further demonstrates a belief that affirmative consent frameworks found throughout campus consent culture belong in our courtrooms.

As sexual assault is a crime of general intent, the Crown only needs to demonstrate that the accused intended to touch the complainant in order to satisfy the mens rea requirement of the offence (R. v. Daviault 1994, referenced in R. v. Ewanchuk at para. 41). However, to avoid convicting those who would be morally innocent, having honestly but mistakenly believed that they had consent, the accused may challenge evidence of mens rea by establishing an honest but mistaken belief in consent (R. v. Creighton 1993; R. v. Park 1995; Pappajohn v. The Queen 1980). Following Ewanchuk, McLachlin C.J. commented that this defence would only be available in instances where the defendant took reasonable steps to ascertain consent, and in doing so, "believed that the

\footnotetext{
${ }^{4}$ Paras "a" and "c" were endorsed by the court in Al-Rawi, however the remaining points were rejected in light of authoritative Supreme Court judgements. In Cuerrier, Mabior, and Hutchinson, the Court rejected the argument that knowing risks and consequences were necessary elements of consent and, in Esau, communicating a voluntary agreement was found to constitute the mens rea of an offence, as well as reasonable steps that may be taken by an accused to determine an honest but mistaken belief. Communication does not make up the actus reus of consent; this must be assessed solely through the complainant's testimony of their subjective state of mind.
} 
complainant communicated her consent to engage in the sexual activity in question" (Plaxton 2015, p. 14, citing R. v. Ewanchuk 1999, at para. 46, emphasis in original). This addressed a great many of the critiques that had been previously levelled at the honestly mistaken belief defence (see Tang 1998, p. 264), as the belief now had to be based in observable, objectively reasonable grounds.

It is, at this point, that we see a shift towards affirmative consent, particularly in light of L'Heureux-Dubé J.'s assertion that “the mens rea of sexual assault is not only satisfied when it is shown that the accused knew that the complainant was essentially saying 'no', but is also satisfied when it is shown that the accused knew that the complainant was essentially not saying 'yes"' (R. v. Park 1995, at para. 39). Here, to establish an honestly held, mistaken belief in consent, an accused must point to an observable, communicated "yes" by the complainant to the specific sexual activity in question. In Michael Plaxton's monograph, Implied Consent and Sexual Assault: Intimate Relationships, Autonomy, and Voice, he comments on the many kinds of casual sexual touching that, in light of Ewanchuk and J.A., would be characterized as sexual assaults under the law (2015, p. 14); here, his argument is rooted specifically in expressions of intimacy between longterm heterosexual couples, but it could easily be extended to other relationships. Plaxton makes the case for implied consent being respected in the law, arguing that this upholds the importance of sexual agency, the idea that sexuality and sexual contact between couples embodies a way of living reflexive of mutuality, and their own normative means of expressing affection (Plaxton 2015, p. 20). 
Sexual contact will naturally look different from couple to couple. More recently, conversations have been centred on the legality and social acceptability of certain forms of consensual sexual contact with respect to harm (see R. v. Welch 1995; Khan 2014a). While this may fall within Plaxton's understanding of mutuality and normative expressions of affection within relationships, it often clashes against the common law. A leading case on sexual contact and harm, R. v. Welch (1995), concerns what the complainant described as a violent sexual assault, involving restraint, hitting, and aggressive penetration of the vagina and rectum. The accused had claimed consent, however the jury was instructed by the trial judge that a defence of consent could not apply considering the nature of the activities. This suggests that, in cases that involve bodily harm, the Crown does not bear the burden of proving non-consent and consent to bodily harm is not valid in law. The decision was upheld in the Court of Appeal, which suggests that, in cases involving bodily harm, there is no available defence for those who are criminally accused (Khan 2014a, p. 4).

R. v. J.A. (2011) further contributed to the common law's pejorative reading of BDSM or non-normative sexual practices in its judgement. Here, the court focused exclusively on the question of "whether consent for the purposes of sexual assault requires the complainant to be conscious throughout the sexual activity" (R. v. J.A. 2011, at para. 21). Ultimately, the Court deferred to Parliament's intention: that consent has previously been dealt with in such a way that there must be ongoing, conscious, and present consent to all forms of sexual activity in question (R. v. J.A., at para. 65). While the Court acknowledged that this may be unrealistic at face value, they deemed it inappropriate to 
find exceptions or ways to make this exercise of common law more practical. As the complainant was unconscious during a period where sexual contact continued, her testimony that the contact was consensual was ignored.

In previous work, I have argued that this iteration of law is built off of risk averse strategies of governance that privilege readings of violence over pleasure (Menzie 2018). A great deal of academic work post $J . A$. has recounted the couple's history of intimate partner violence in order to overlook testimony provided by the complainant (Gotell 2012; Craig 2014; Busby 2012; Koshan 2016). In fact, the Women's Legal Education and Action Fund's (LEAF) intervening factum focused almost exclusively on the context of intimate partner violence, rather than acknowledging the complainant's testimony of consensual kink (Khan 2016c; Menzie 2018). However, accepting an iteration of law that attaches a greater importance to readings of violence over readings of agency and kink is problematic. Again, we reach the same juncture in Ewanchuk, where, in law, both the context and perception of fear and violence are privileged above readings of pleasure, attractions, and agency. By requiring affirmative consent, the law privileges risk-averse policies over non-normative pleasure. Communicational nuances are ignored, and sexual scripts are forced into a universal framework. We also further establish a disregard in law for the legitimacy of sexual practices that fall outside the norm.

In Welch, Ewanchuk, and J.A., the test for establishing sexual assault prioritizes risk aversion over non-normative pleasure and puts a particular kind of burden on the defence. Consent in law, and in practice, is determined through a person's subjective state of 
mind. With respect to sexual assault, implied consent is not a valid defence and there is no legal consideration of objectively present contextual cues that would signal consent to an accused. Above, I outlined the three components of the actus reus for sexual assault: (1) touching; (2) of a sexual nature; (3) without consent. This legal framework criminalizes the act of sexual touching where consent is not present, but provides no objective standard for consent and relies solely on the complainant's subjective state of mind. The complainant may even give objectively visible cues that indicate consent, however the accused will still be found guilty of the actus reus (R. v. Ewanchuk 1999). In Ewanchuk, the complainant was found by the trial and appellate court to have given objectively visible contextual cues that she was consenting to sexual touching. However, the court found that these cues, while present, could not constitute consent because of her subjective state of mind, because she was afraid. While I am not suggesting that complainants be held accountable for actions taken in the interests of self-preservation, the decision to overlook objectively present cues sets problematic legal precedent. Effectively, a subjective - and possibly unfounded - feeling of fear is prioritized over an objectively present and reasonable reading of sexual interest. This test restricts the ways we might communicate sexual interest and proposes instead an explicit, ever-present bureaucratic framework relying heavily on caution and mistrust. It further exceptionalizes sexual offences, ${ }^{5}$ and imposes an evidentiary burden on the accused to defend themselves should they have misread the complainant's subjective state of mind.

\footnotetext{
${ }^{5}$ Indeed, implied consent has been found to be an appropriate legal defence to causing harm in other circumstances, for instance, surgical interventions, and injuries sustained during sports or stunting $(R$. $v$. Cey 1989; R. v. Leclerc 1991; R. v. Jobidon 1991).
} 
An accused charged with sexual assault has available to them a defence of an honest but mistaken belief in consent. This belief cannot arise from self-induced intoxication, recklessness, or wilful blindness, and further, the accused needs to demonstrate that they took reasonable steps to ascertain that the complainant was consenting. This is captured under section 273.2 of the Criminal Code, which reads as follows:

273.2 It is not a defence to a charge under section 271,272 or 273 that the accused believed that the complainant consented to the activity that forms the subject-matter of the charge, where

(a) the accused's belief arose from the accused's

(i) self-induced intoxication, or

(ii) recklessness or wilful blindness; or

(b) the accused did not take reasonable steps, in the circumstances known to the accused at the time, to ascertain that the complainant was consenting

Here, paragraph (b) departs in some ways from the common law. Paragraph (a)(i) captures the common law rule that sexual assault cannot be defended or justified through self-induced intoxication, and paragraph (a)(ii) reflects the Court's judgement in $R . v$. Sansregret (1985). However, paragraph (b) introduces an objective standard in determining whether the accused's belief was honestly held. Their state of mind is assessed alongside actions taken to support or reinforce their belief in consent. This also has the effect of placing a tactical or evidential burden on the accused to introduce evidence that is capable of raising reasonable doubt to their intentions and speaking to an honestly held belief in consent. The Court's disparate application of standards here is important. An accused must provide objective evidence to their state of mind should they honestly hold belief in consent, where a complainant's subjective state of mind is sufficient. Fear and pleasure are assessed and weighted differently by the Court, and 
greater scrutiny is applied to those who are accused under criminal law prior to conviction.

This legal test is demonstrative of an argument made many times before in sociolegal and criminological work: that social life is governed by risk (O’Malley 2004). The Court here, in many authoritative judgements has ruled that fear of violence must take precedence over spontaneity and, potentially, pleasure. By stipulating that fear may be unfounded, while contextually present cues indicating consent still must be asserted through a communicated "yes," the Court has rooted this governance of risk in precautionary logic (R. v. Ewanchuk 1999; R. v. Park 1995; Haggerty 2003). Risk of miscommunication in these contexts is incalculable, which thereby forces the courts to rely on intangible and unmeasurable anxieties surrounding sexual contact and expression (Haggerty 2003; Jochelson, Gacek and Menzie 2018). The Supreme Court of Canada's framework for consent may be better equipped to address harm, but this net has been widened on the basis of a harm that is impossible to measure, and effectively privileges risk-aversion over non-normative pleasure.

Theoretical Section: Harm, Judging, and Belonging

Past scholarly work has examined the legal denunciation, censorship, and criminal sanctions imposed on other forms of sexual expression - such as pornography and sex work - in the context of a changing conception of harm within neoliberalism (Jochelson and Kramar 2011; Jochelson, Gacek and Menzie 2018). Neoliberalism, and the resulting 
imposition of legal censorship and criminal sanctions, is credited as using both power and subjugation to create sexual subjects (Jochelson and Kramar 2011, p. 9). With respect to consent, I would suggest that there is a similar shift towards a new conception of harm that justifies legal interference into the sexual lives of citizens.

Past scholarship has illustrated that neither consent nor harm can be considered selfevident categories within the law (Khan 2014b, p. 428). In not being observable or measurable phenomena, these concepts are malleable, and may restrict or make space for certain behaviours over others as the common law evolves. This understanding is explored by Jochelson and Kramar's theoretical conception of law's “mutant harm principle," applied then to the regulation of obscenity and indecency in Canada (2011). Here, they argue that criminalization is not confined to a single logic and, through these transmutations, the law is able to curtail many liberal critiques that would otherwise be applied to an unjust exercise of power. John Stuart Mill's On Liberty chronicles the legitimate exercise of state or societal powers upon individuals, arguing that "the only purpose for which power can be rightfully exercised over any member of a civilized community, against his will, is to prevent harm to others; his own good, either physical or moral, is not a sufficient warrant" $(1859$, p. 13). However, by choosing to frame harm to others as not always physical but, rather, as potentially an intangible harm to the community, the court is able to cloak their enforcement of a more conservative moral order within the terms of the "proper functioning of society" (Jochelson and Kramar 2011, p. 16-17). Jochelson and Kramar argue judicial decision making in these contexts 
has removed voices of certain sexual communities in order to describe what constitutes bad sex and, by extension, what must then be seen as good sex (2011, p. 17).

With respect to consent and the cases outlined earlier in this chapter, the law has deemed bad sex as sex that leaves marks, causes pain, or relies on consent that is implied or given in advance (R. v. Welch 1995; R. v. Ewanchuk 1999; R. v. J.A. 2011). Consequently, while not explicitly addressed in Canadian statutory law, BDSM practices are often captured by case law as violent, pathological, and harmful, resulting both in stigmatization and sometimes criminalization through various legal forums (Khan 2014c, p. 225). In Vicarious Kinks: $s / m$ in the Socio-Legal Imaginary, Ummni Khan examines the legal and societal anxieties surrounding s/m practices. Considering challenges posed both by queer theory and risk-aware consensual kink (RACK), Khan notes "that the process of deciding which risks and desires will be considered unacceptable, and which will be ignored or naturalized, depends on sexual ideology that privileges vanilla risk aversion over non-normative desire," regardless of expressed consent (2014c, p. 259).

Decentering BDSM specifically from this inquiry, many normative expressions of sexual attraction and desire are also captured under sexual assault legislation that fails to account for implied or advanced consent. This was raised by the Crown in J.A., as it was noted that many couples will kiss their sleeping spouses as an innocent expression of affection and that there may be just cause to exempt certain behaviours from the criminal law's reach. Implied consent is also noted as quite common amongst long-term romantic partners who may choose to allow for spontaneous kissing or light sexual touching in 
order to demonstrate affection (Plaxton 2015). In this sense, our legal framework is both discriminatory and overbroad: discriminatory in that many common law decisions criminalize and stigmatize BDSM sexual activity, and overbroad in that the potential exists for egregious prosecution of what many consider to be blameless sexual contact.

The purpose of this thesis is not to scour case law and courtrooms for examples of discriminatory or overbroad prosecutions. However, this discussion is necessary when we account for functions of criminal law aside from punishment. In articulating consent frameworks that contest both normative and non-normative sexual contact, legislation could be said to lose credibility and authority in the eyes of the public: it no longer serves as a reliable moral guide (Plaxton 2015, p. 27). Beyond this, by labelling what many consider to be innocent conduct as criminal, the expressive function of criminal law over extends its boundaries, and either delegitimizes itself or the behaviour in question.

It could be suggested that, in our current social climate, this expression of law is most likely to condemn certain sexual practices rather than impact the public's faith in criminal law. It is not a novel observation that dominance feminists have maintained that certain forms of sexual expression bring about detrimental consequence to women in society and equality writ large, thereby establishing a defensible moral position in avoiding harm (Benedet 2001; Cole 1989; Dworkin 1998; MacKinnon 1987; Mahoney 1991). This is often expressed through anti-pornography feminism but, under an emerging societal context of anti 'rape culture' activism, it is unsurprising that similar moral positions have been advanced with regard to forms of consensual sex as harmful to women and equality. 
Indeed, university campuses have increasingly been used as sites of consent education and awareness, where students are educated in reading affirmative, "legitimate" consent (Sheehy and Gilbert 2015; Endrapeoncampus.org). Activity that takes place outside of these frameworks is to be understood as non-consensual and a form of sexual violence. Thus outside of the law, in many facets of society, we are being told the process for reading and, conversely, granting consent, and are being instructed that behaviours that fail to fit within this framework are sexually assaultive.

In the introduction of her book, The Rape Victim, Elaine Hilberman writes that rape is "the ultimate violation of the self, short of homicide, with the invasion of one's inner and most private space, as well as the loss of autonomy and control.” (1976, p. x) As a clinician, Hilberman's book serves to inform medical practitioners and those who work closely with victims to the grave, substantial harm that rape causes. Hilberman also points to the work of many dominance feminists and a failure in law to explain and critique the pervasiveness of rape in society. This conception of rape, as an ultimate violation, has since mobilized outside of clinical and feminist work. Most recently, in reading from his sentencing decision $R$. v. Ururyar (later overturned), Justice Zuker chose to describe rape as "the ultimate violation of self, short of murder, except that it can be the murder of a soul" (DiManno 2016) ${ }^{6}$. Our understanding of sexual assault as an attack on identity has not changed since the 1970s. It is noteworthy that both Hilberman and Justice Zuker chose to categorize rape this way: as "the ultimate violation," "short of

\footnotetext{
${ }^{6}$ Justice Zuker used this language at a press reading of the decision; while a similar imagining of the rape victim was expressed often throughout his judgement, he did not refer to rape as "the murder of a soul" in the actual case.
} 
murder"; it is further interesting that Justice Zuker went beyond this still, characterizing rape as "the murder of a soul". He suggests that moving past this experience is impossible. Rape is socially and legally encoded as a life-changing event: one that cannot be ignored, but perhaps can be prevented should we adopt an institutionally mandated, stringent, and affirmative consent framework that responsibilizes us as citizens to both maintain our bodily integrity and avoid legal repercussions.

Citizenship has been defined as a "set of practices which constitute individuals as competent members of a community" (Turner 1994, p. 159). Sexual citizenship began with the theoretical premise that a society makes space for acceptable sexualities and practices and, in the process, excludes queer communities. Past sexual citizenship literature (Bell and Binnie 2000; Richardson 1998) has argued that citizenship is heterosexual, and that technologies and practices of sexual citizenship are intended to push them out or make them sexual strangers within body politic (Phelan 2001). Cossman's Sexual Citizens: The Legal and Cultural Regulation of Sex and Belonging built off of this theory: rather than centre her argument on sexuality alone, she argued we use various technologies hand in glove to become citizens and affirm our belonging through both sexuality and identity (2007). Cossman also theorized that, much like gay and lesbian subjects, straight subjects also constituted and reconstituted identity with the possibility of unbecoming citizens (2007; see also Plummer 2003). Consensual sexual practices are subject to regulation; for Cossman, this is predominantly seen through the legal and societal governance surrounding sexual speech, pornography, sodomy, and sex 
toys, and how this governance operates in order to produce or deny sexual citizenship (2007).

Cossman states that the new process by which sexual citizenship is formed is governmental and self-disciplinary (2007). Under neoliberalism, many scholars have pointed to a new form of governance that, rather than relying on direct state intervention, prompts the self-governance of its citizens (Dean 1999; Hunt 1999; Rose 1999, 2000; Rose, O’Malley and Valverde 2006). While past work has imagined citizenship in the public sphere to be predicated on appropriate sexual practices in private (Cossman 2007, p. 6), understanding how choice and self-regulation "makes" a citizen unsettles the idea of firm, state-enforced boundaries. Sexual citizenship should be understood as "an ambivalent practice, simultaneously subversive and disciplinary" (Cossman 2007, p. 9; Stychin 2003). When we consider the dominant social construction of rape as the most destructive act, and one that foundationally changes its victims, it is hard to see citizens choosing to subvert this boundary and reform citizenship on their own terms. Rather, to maintain citizenship, subjects may prioritize risk-aversion and precaution across their sexual interactions (Cossman 2007). This may manifest in anxiety over past sexual interactions for some individuals who, after having evaluated an ambiguous experience that they initially believed to be consensual, finds that the "consent" does not match up with an institutionally governed and defined consent framework.

It is not my position that affirmative consent frameworks are ineffective; rather, I would suggest that consent is a personal process. It becomes dangerous when consent, the only 
piece separating consensual sex from sexual assault, is institutionalized, disciplinary, and enforceable, while the voices of consenting parties are minimized or ignored, as in J.A.. Here, the role of law in establishing (non)consent should not be viewed exclusively as repressive, but also as disciplinary and constitutive (Stynchin 2003). By taking a personal, affective decision and subjecting it to legal rules changes the foundational aspects of the act. Legal and social safeguards that are in place today requiring partners to obtain consent according only to a set framework paradoxically suggests that: (a) partners who anticipate or presume consent outside that framework are risky, and potentially assaultive, partners; (b) consent not given under that framework is untenable.

Institutionalizing consent in this way calls for both those reading and granting consent to accept a one-size-fits-all framework and, in the process, ignore their own agency and autonomy. In the same sense that it is inappropriate to rely on rape myths to establish consent, it is inappropriate to rely on law to speak for potential victims. If we accept that consent is still a personal process, and not an institutional one, then it would be entirely possible to grant and read consent outside of this framework (indeed, Plaxton argues this is the case within long-term relationships). However, as this framework continues to pervade public discourse and gain legitimacy, I argue that we will increasingly see individuals doubting or questioning the legitimacy of their consent or the consent of their partners.

As issues surrounding consent become increasingly confused and contested, I argue that some individuals will seek out advice and judgement within their communities to help them both self-regulate and manage risk. By soliciting third-party advice and judgements 
online, individuals may be able to find clarity into how their (non)consent would be socially understood. By venturing online, individuals are actively engaged in the construction of the narrative. When analyzing my data, it was particularly interesting how posts and comments echoed legal structure: the title typically honed the focus on whether assaultive conduct took place, the body of the post contained a detailed fact scenario, and very few posts focused on subjective feelings and experiences, and rarely inferred thoughts on their partners. Comments were predominantly centred on addressing the main question of assault, and comments that received the most approval from the judging community were listed first, with dissenting comments following. I would suggest that the choice to bring private sexual experiences to a public, albeit anonymous realm, is a form of risk management. Whether OP is concerned with being assaulted or assaultive, they are reaffirming their status as citizens by attaching importance to the social and legal meaning of their experience: there is a recognition that being sexually assaulted has a fundamental impact on your personhood, and that being found to have committed sexual assault comes with substantial reputational harm and disciplinary consequences.

The familiarity and self-assurance associated with judging these narratives is not a new phenomenon. The expertise needed to judge has, in many ways, already been cultivated through the indoctrination of law through popular culture (Kamir 2006; Asimow 2006). Kamir's Framed: Women in Law and Film analyzes the interrelated discourse of law and film, and provides a theoretical foundation on how law and film can be read in a socially meaningful manner (2006, p. xxii). Kamir notes that "films, much like judicial decisions and legislative rhetoric, can - and do - constitute communities (of viewers) that are often 
engaged in judgment, pseudolegal reasoning, the pursuit of justice, and a corresponding self-fashioning" (2006, p. 3). Viewers are afforded a sociocultural persona and invited to become part of an imagined judging community; judging in virtual space rather than cinematic space poses no substantive procedural differences and, in fact, may even allow for more user confidence due to a perceived familiarity with the legal system.

Law exercises its rule through expression and education, as well as through culture. Most people learn legal systems and procedures through popular culture, and are often unaware that they are being socialized to a certain belief system and receiving a form of popularlegal education (Kamir 2006). Western audiences are much more familiar with Hollywood's dramatized portrayal of the common-law legal system, and often conflate this portrayal in place of their own legal systems and traditions (Machura and Ulbrich 2001). This often becomes the starting point for a users' understanding of law and precedent, along with social convention and teaching. Kamir comments on the social value and effect of law films, arguing that they "train audiences in the active execution of judgment while examining - and often reinforcing - legal norms, logic and structures" (2006, p. 3). Construction of subjects and communities within a legal text or film become inseparable from the judgment process, which Kamir argues creates a large scale "legal indoctrination," allowing viewers to cultivate expertise (2006, p. 3).

Borrowing from Kamir's theory on cinematic jurisprudence, I suggest that many of the themes she identifies are salient and applicable to a "virtual jurisprudence," observable through the facilitation of virtual trials online. Referencing the field of narratology, 
Kamir examines the explicit and implicit mechanisms that constitute an "implied reader/viewer" (2006, xvi). Hosting a virtual trial necessitates participation from a broader community; readers are therefore implied and accounted for within the text "in the very rhetoric through which [they are] required to 'make sense of the content'" (Rimmon-Kennan 1983, p. 117). Here, the construction of text matters in part, such as how the post is titled or how the actions are described, but, perhaps more critical is a user's choice of virtual space. It is intuitive and can be assumed that users may find different answers soliciting a BDSM affiliated community (r/bdsm or r/BDSMcommunity) versus a relationship advice community (r/relationship_advice). The "implied reader/viewer" can be conversely considered against the real viewer; they may be unresponsive or resistant to the invitation behind the text or may challenge and subvert the underlying intentions of the author. Real viewers may instead interrogate the purpose of asking these questions, challenge the fact scenarios outlined, or may even choose to stay "offline" and not participate. Posts that elicit a virtual jurisprudence may be intended as "trial runs" before electing to report, as a few users had noted that widespread validation on Reddit persuaded them to access more traditional forms of justice. In this sense, these spaces are similarly a product of risk-management due to the stigma surrounding accusations of sexual violence and the likelihood of revictimization through the legal process.

\section{$\underline{\text { Conclusion }}$}


This project suggests that, in light of dramatic legislative shifts and attempts to institutionalize a universal consent framework, the boundaries between consensual and assaultive sex are being redefined, and for some, becoming blurred. Individuals are able to place less trust in themselves and how they perceive sexual interactions, and less trust in their partners. In order to navigate grey or ambiguous sexual contact, many choose to seek a virtual jurisprudence in lieu of or in advance to a formal legal response.

Ultimately, these changes in legislative practice and in consent education stigmatize risky sexualities and teach citizens to become increasingly risk-averse. These spaces are a product of the many anxieties that now surround sex, particularly when consent has been placed under a microscope. In attempting to overhaul the way that people grant and read consent, law and society has pushed citizens to peripheral systems, like Reddit, to make sense of their experiences. This project analyzes renegotiations of consent arising from these spaces to demonstrate the effect of societal and legislative governance and to understand how users construct and benefit from virtual trials. 


\section{Chapter 3: Methodology and Space}

This chapter will discuss the methodological approach used to conduct an ethnographic content analysis (ECA) of the data collected from Reddit. My logic behind using ECA, as well as my approach to data collection, thematic coding, and the analysis of the virtual trial process manifesting on Reddit will then be justified. Reddit will be discussed as a space of inquiry; there is limited academic work analyzing Reddit as a research site, however some scholars have attempted to theorize the emergence and nature of this virtual community. It will also outline the affordances of Reddit and how this influences the discussions that take place, specifically with regard to anonymity. Unlike many other forms of social media, Reddit manages to maintain anonymity in its user base and avoid context collapse. This makes these discussions unique as Redditors are aware that they are petitioning strangers for advice within a shared online community. This discussion will be supplemented with some observations that occurred throughout my analysis. Finally, I will outline some theoretical considerations that informed my analytical approach, points of inquiry, and treatment of data and subjects. As both space and method are necessarily interrelated, at points, this discussion will overlap.

\section{$\underline{\text { Ethnographic Content Analysis }}$}

Hammersley and Atkinson described the value of ethnography as being most visibly apparent in relation to the development of theory (1983). Ethnography lends itself well to challenge preconceptions from both literature and popular academic thought: once immersed in a community, it is difficult for a researcher to maintain a theoretical stance 
that is contested by the people and the space of inquiry (Hammersley and Atkinson 1983, p. 23). It is here that we can see that immersion for the purpose of conducting ethnographic work is critical. Traditional ethnographic work had involved participation, “overtly or covertly, in people's daily lives for an extended period of time, watching what happens, listening to what is said, asking questions," with the intention of collecting as much data as possible (Hammersley and Atkinson 1983, p. 2). However, contemporary society has allowed for the examination of less conventional spaces with the aims of understanding culture. Ethnography is becoming a more malleable practice and more of an orientation and awareness of research rather than a concrete method. To research ethnographically is to acknowledge that participant observation is present, directly or indirectly, in every research project through topic selection, method of study, data collection, analysis and interpretation (Altheide and Schneider 2013, p. 23). The writing of culture today may rely on analyses that go beyond physical space, that call upon researchers to think more critically about the role of the internet and digitization as they create novel sites of inquiry, production, and possibility (Fortun 2010, xix)

As Kim Fortun writes in the foreword to Writing Culture, today, it is all about technology. These spaces are becoming the platforms "on which thought and life unfold," and are deserving of our attention (Fortun 2010, xx). The conventions of ethnographic observation, however, change when the space is virtual rather than physical. Since its genesis in 2005, Reddit has grown into one of the largest online communities, one that has demonstrably evolved from a gateway of extant web content to "an increasingly diverse, self-referential community that focuses on and reinforces its own user-generated 
content" (Singer, Flock, Meinhart, Zeitfogel, Strohmaier 2014, p. 518). Reddit's

maturation has not only resulted in its widespread popularity but in a broad user base that allows the site to host these discussions with a new, dynamic public that attempts to artfully navigate questions of consent and sexual violence and provide the $\mathrm{OP}$ with insight. Reddit as a particular space of inquiry has been described using Bahktin's formulation of "carnival": it is grotesque and chaotic, compelling and repulsive (Massanari 2013; Bhaktin 1984). A single thread may contain a wide-ranging, disparate body of content including in-group references, puns, animated GIF responses, images and stories, links to video content, racist and sexist speech, put up against sincere, meaningful commentary and dialogue (Massanari 2013, p. 2). My inquiry, particularly with respect to sexual consent and the construction of virtual trials, is concerned with content and searching through the "carnival" to decipher meaning and intention. In attempting to make sense of these discussions and logical underpinnings, however, immersion is necessary to the space; it is also foundational in developing cohesive theoretical contributions that do not simply echo conventional assumptions surrounding sexual assault and gender-based violence in academia and law.

Balancing the need for community immersion and attention to content is best achieved through an ethnographic content analysis (ECA). Altheide and Schneider (2013) explore ECA as a research method most recently in the second edition of Qualitative Media Analysis. Here, they further develop the framework outlined by Altheide (1996) to include examinations of social media and user-generated content. They reinforce the idea that ethnographic research requires immersion within the contexts, environments, 
situations and lifeworlds of its subjects (Altheide and Schneider 2013, p. 24). However, in cases where "the meaning of an activity remains paramount... ethnography can also be considered as a methodological orientation independent of a specific subject matter" (Altheide and Schneider 2013, p. 23). In this sense, completing fieldwork means critically exploring the content of interest to research questions. While I am unable to follow subjects as they pass to and from various threads and subreddit communities, I can immerse myself within the sites of inquiry and devote time to understanding how they operate, regulate, and allow for user participation.

Reddit can be conceptualized as both one and many cultures and, more recently, "a community of communities" (Massanari 2015, 2017). Here, a user's choice to host consent negotiations within a specific virtual space can provide contextual cues for how they identify, where they find belonging, or how they view their experience. Singer et. al's (2014) longitudinal study of Reddit found a growing diversification of these communities, which Reddit labels as subreddits (identified by $\mathrm{r} /$ ). Guided by the need to immerse myself within the research setting, I initially selected six subreddits, which I later reduced to five. Prior to collecting data and throughout the data analysis process, in order to immerse myself in the virtual spaces of concern, I routinely visited and read content from: r/sex; r/relationships; r/relationship_advice; r/bdsm; r/BDSMcommunity. In the interests of capturing the nature of each space, I would spend five hours every week reading through posts. ${ }^{7}$ I limited my immersion to one subreddit per day to avoid

\footnotetext{
${ }^{7}$ Reading through posts included content that was not specific to my inquiry of virtual trials and (re)defining sexual experiences. Immersion was used mostly to understand the context and space from which discussions emerged, and whether these specific discussions were treated differently than others within the same communities.
} 
confusion (i.e. on Monday, I would spend an hour reading through posts on $\mathrm{r} / \mathrm{sex}$, on Tuesday, an hour on r/relationships, etc.). ${ }^{8}$

There are numerous benefits to limiting the area of inquiry to specific, targeted subreddits Subreddits can be restricted to those that may be intuitively chosen: specifically, those that demonstrate a strong link to sex, sexual practices and identities, and interpersonal relationships. This ensures that: the subreddit regularly engaged in discussions on sex and consent; comments came from Redditors who are familiar and comfortable discussing sex or relationship dynamics; community guidelines allow these discussions. Similarly, certain subreddits that label themselves under a specific political ontology (i.e. r/TwoXChromosomes) or subreddits that suggest a degree of certainty by users (e.g. $\mathrm{r} /$ RapeCounseling and $\mathrm{r}$ /SurvivorsofSexualAbuse) are also easily avoided. Outside of these concerns, it allows for thorough immersion and data collection within the space without being hindered by time restraints. Posts from private subreddits can also be filtered out from inquiry; some scholars have suggested that this alleviates any ethical or methodological concerns that may arise from seeking access online for the purpose of research (Altheide and Schneider 2013). While this perspective may not be universally accepted, Reddit is unique from other online spaces and forms of social media. This space affords its users additional protections should their posts or comments be used in research. Redditors may choose to provide their email address when making an account, but they do not provide a name, birthday, or other identifying information that might be

\footnotetext{
${ }^{8}$ This level of immersion was maintained until January 2018. Immersion was difficult to continue at this level once I began writing, but I would routinely scroll through the front pages of each subreddits' "hot" posts about once a week to maintain familiarity.
} 
found on other social networking sites such as Facebook or LinkedIn. Further, if an email address is provided, it is not linked publicly to the account. Nearly every post used in this analysis came from a throwaway account, where the OP created the initial post and then conceivably abandoned the account. Finally, Redditors do not 'follow,' 'friend,' or otherwise maintain relationships in these spaces; the only way to interact with another specific user is if they have provided their username and are sought out. In light of this, Redditors are aware that they are making their information visible to strangers. In fact, they are engaging in a call to response for others to impartially consider their own experiences. This project has attempted to further ensure the privacy of users directly cited in this thesis by consulting their posting history and confirming that they have not provided direct or indirect identifying information.

\section{$\underline{\text { Data Collection and Analysis }}$}

Online spaces, including Reddit, host a massive amount of content that grows larger every day. With respect to researching these spaces, the vast amount of available data has been identified as a potential hindrance to the data collection process (Altheide and Schneider 2013, p. 108). With the intention of being mindful of timing, yet thorough, my research process was informed by guidelines for collection and inclusion of data involving set requirements and reflexive note-taking. I confined my research to one subreddit at a time in order to "mine" or collect all relevant posts before moving on. This reinforced immersion as I was reading, identifying, and reflecting on how narratives were received and influenced by subreddit rules and moderators. My timeframe was restricted to posts that were made from January 2010 up until January 2018. This ensured that these 
discussions would be located temporally near the sociolegal reimagining or redefining of consent to its current iteration, and also allowed time to complete the analysis. The posts collected would therefore be most likely to engage with the tensions discussed in the theoretical body of this thesis: that law and society may be articulating a standard or framework for consent that is unrealistic or even impossible (Khan 2014a; Plaxton 2015; Menzie 2018). Posts were collected and stored for analysis if: the OP was asking about their own sexual experience, rather than a hypothetical experience or the experience of a third party; a fact scenario is provided; the post received at least one comment; I had no reason to question the legitimacy of the post or the OP; ${ }^{9}$ the post is publicly available. Guided by this collection criteria, I selected 66 original posts on Reddit, including over 4,000 comments, for analysis.

Data that has been recovered online can be reviewed as a "live" website, or saved and stored (Altheide and Schneider 2013). For the purpose of this project, data collected from Reddit was stored in a qualitative software program (QSR NVivo) for analysis; the website was also saved as a PDF file so that data would be recoverable and not at risk of being deleted or modified. A recommended best practice for ECA research in online spaces also requires the routine revisiting of past sites of inquiry to view new comments, updates, or new posts (Altheide and Schneider 2013, p. 8). To accomplish this, I routinely revisited subreddits and past posts while in the analysis stage of my project in order to

\footnotetext{
${ }^{9}$ This was intended to account for the practice of "trolling" online. Trolling has been observed in many online communities, including Reddit (Bergstrom 2011). Here, I gave deference to the community. While a few posts received comments that accused OPs of trolling, they were always in the minority and received significant disapproval reflected in their karma points. There was also no evidence that OPs had lied or intentionally misrepresented elements of their narrative when they were accused of trolling.
} 
remain open to discovery of new cases and challenges to my theoretical framework. However, I did not include any posts that were made on or after January of 2018 in the interests of time.

After concluding data collection and maintaining immersion within subreddit communities, I began to code using an integrated approach with both deductive and inductive methods. My first concern was with the comments I could expect to see emerging from these posts, which I chose to separate generally by the Redditor and the OP. This resulted in the creation of three nodes under Redditor comments ("affirming an assault," "denying that an assault took place," and "unclear/grey area") that would weigh in on whether an assault had occurred. Affirming and denying were used with respect to the potentially assaultive conduct and not with the OP's positionality to refocus the inquiry on the act. I also began with three nodes examining the OP's comments ("clarification," "additional questions," and "judgement rendered") so as to collect the most information possible about the activity in question, their engagement with Redditors, and how/whether they chose to reframe their experience. As my inquiry progressed, I saw the need to include new nodes to categorize comments that went beyond the act and initial judgement, such as "victim blaming," "consent education/consent frameworks," and "external solution proposed" which was later branched into five more nuanced nodes ("cops/reporting," "therapy/medical," "violence," "legal remedy," and "prison"). Initially, coding was done deductively and to further familiarize myself with the data to generate inductive codes. After completing first-level coding, data was revisited and coded again using the generated inductive codes. Finally, a 
third-stage of coding took place to ensure consistency and reliability, and to bring a strong familiarity with the cases and the rules or guidelines that underlie the process.

Using this approach allowed my coding to be both "data-driven" and "template" based; an inductive approach brings flexibility and allows for surprises and an open inquiry, while the deductive process centres the research question and conceptual framework within the coding process (Fereday and Muir-Cochrane 2006, p. 82-83). An integrated approach navigates the limitations inherent in both forms while still maintaining the benefits. Despite the fact that there is no academic treatment of these posts, it followed that Redditors would be prompted to address the question asked of them, and it was apparent during data collection that the OP would occasionally resurface in the comments to provide clarification, ask additional questions, or provide insight into how they have come to view their experience. Here, deductive coding was useful to integrate "concepts already well known," which allowed for a more critical understanding of past insights, and referred back to the initial research questions (Bradley, Curry and Devers 2007; Elo and Kyngas 2008). Deductive coding is fallible in that it forces the data to fit within specific codes and limits the potential for growth. Data that is coded inductively is able to develop from the ground up, and may be able to better reflect the concepts within the analyzed data (Bradley, Curry and Devers 2007). However, a purely inductive approach may subsume the researcher in the themes of the data and cause them to lose sight of the initial point of inquiry. An integrated approach allowed for a thematic and conceptual mapping of these spaces, left my analysis open to challenge and surprise, and maintained a close tie to the initial questions that prompted this work. 


\section{$\underline{\text { Reddit as a Space of Analysis }}$}

Reddit, despite receiving very little academic treatment, has been described as "carnival," as participatory play or a "game," as a social voting site, as 'dude space' (said otherwise, a misogynistic, hostile environment) or, perhaps more technically, as a link aggregation website with a comment section (Massanari 2013, 2015, 2017; Gilbert 2013; McClung Workman 2014; Zuckerman 2012; Bergstrom 2011). While these conceptions are not inaccurate, they make assumptions about how the space is primed to operate and lose sight of a user's agency to make the space their own. Imagining Reddit as a "game" or a social voting site ignores the activity of those who may choose not to play. Similarly, understanding the space as hostile, or as "carnival," brings the precariousness and instability of the space to the forefront; not only is Reddit understood here as a site of oppression and danger, it is also understood as wayward and unpredictable. Despite the blending of media, my extensive immersion brought about a comfort in the messiness. The use of GIFs, links, photos, and comments were not a short-sighted or sloppy engagement, but were instead often deep, nuanced, and a critical connection and commentary to the original source material. The mixing in media was demonstrative of conscious and intentional shifts of engagement: many users would post links to content on YouTube reminiscent of the OP's fact scenario, or would post links to educational spaces in order to help the OP regain agency and define their experience on their own terms. Conversely, I found Reddit, specifically the posts connected to my research question, highly organized and technical. 
My choice to imagine these discussions as virtual trials came from this structure. Every post I uncovered followed near identical formats despite emerging from different subreddit communities with their own cultural codes and rules. Posts would begin with the title, which nearly every OP chose to label after the act: "Was I raped?," "I think I was raped," "Not sure if I was raped?," were all common and predictable indicators that the post aligned with my research. A small subset chose to label their posts by focusing on the grey nature of the contact and their own confusion: "Confused how to feel about something that happened months ago," "Grey areas of consent," "This happened a couple weeks ago and has messed with my mind," were used less often, but still categorically identified the call to answer and the need to make sense of a potentially assaultive experience. In the body of the post, OP's would then begin by laying out a fact scenario. They would identify whether there was a pre-existing relationship with the other party, their mental state throughout, and how they feel about the experience now. Comments would follow where Redditors would attempt to unpack the experience for the OP, often as unbiased third parties. The focus was almost always on the act itself, with some questions asked of OP to clarify the fact scenario that was previously laid out. Many Redditors would also root their analysis in traditional jurisprudence, establishing law as the ultimate answer and authority; OP was often asked where they lived in order to refer back to the jurisdiction. Other Redditors chose to self identify: as victims/survivors of sexual violence; as legal professionals; as trauma counselors or other psychiatric medical professionals; even as rapists, or owning up to previously committing or attempting assaultive contact. However very few comments strayed from the initial purpose of the post, challenging Reddit's image as oppressive or otherwise messy space. 
Similar to current practice with regard to Supreme Court judgements, Reddit's algorithms stack the judgements or comments by majority approval. By this, I am referring to Reddit's social voting function, where users are able (and perhaps expected) to upvote and downvote posts and comments to provide order. Effectively, they separate "good" content from "bad" content as service to other users (Gilbert 2013; Weninger, Johnston and Glenski 2015). The choice to then move beyond the majority opinion and look at dissenting comments is questionable: analyzing entire posts is time consuming, and challenges the authority of the community where OP has placed their trust. However, Reddit's voting algorithms, while highly effective in ordering newsworthy or interesting posts, are questionable in establishing a majority judgement. My decision to analyze posts in their entirety, and to give no particular weight to posts by their karma or value was informed by three reasons. Collective judgements by a community have demonstrable accuracy when compared against individual judgements, even the judgements of experts, however, this becomes untenable within social voting sites like Reddit (Galton 1907; Surowiecki 2005; Weninger, Johnston and Glenski 2015). First, collective judgements on Reddit are questionable because of underprovision or, put simply, users who do not vote. Gilbert (2013) found that, unlike many other large online communities, Reddit has a visibly large rate of underprovision where votes are either misattributed or comments are overlooked in the voting process. Second, voting results may be easily manipulated and narratives may be controlled by Redditors who are able to vote first. Weninger, Johnston and Glenski (2015) found corresponding positive and negative herding effects when they introduced single random upvotes and downvotes to 
posts. Users are swayed by the visible community approval or disapproval that came first, which underscores the need for counter measures to assess community values in a more holistic way (Weninger, Johnston and Glenski 2015, n.p). Finally, placing authority in the raw score ignores potentially contentious posts (ie. a post with a raw score of 200 upvotes may have received 700 upvotes and 500 downvotes; naturally, this is less authoritative than it initially appears). These issues in blindly trusting the majority opinion called for a deeper, textual reading, which was a contributing factor in choosing my methodology. ${ }^{10}$

The upvote and downvote scoring system on Reddit could be said to compromise the authenticity of these spaces and the data my analysis draws from. Reddit members who post or comment on content are subject to this system, and both upvotes and downvotes are quantifiably measurable and tied to a user's reputation. "Upvotes are measured in karma points, and signal to the community that a particular comment of post is substantive and adds to the larger conversation” (Massanari 2013, p. 2). Similarly, downvotes will result in the user losing karma points. For users invested in their online reputation, it could be said that posts like this are subject to impression management and may be censored, altered, or entirely fabricated. However, from my immersion in the space, I would suggest that the risk of this is minimal, and is outweighed by the value this analysis can offer.

\footnotetext{
${ }^{10}$ Although caution should be exercised in assessing a comment's community approval solely through its raw score, I elected to include the raw score or "points" assigned to each comment that I cited in entirety. Each comment referenced in this thesis was assessed qualitatively to be representative of at least a popular opinion on the thread. I made this choice as these are likely to be the comments that speak most clearly to the nature of the space and how grey areas of consent are judged retroactively.
} 
Reddit is unlike other forms of social media in that users are not bound and limited to one virtual presence. Creating an account only requires a username and password; while Reddit will prompt new users for an email address, they do not need to provide any identifying information to make an account, and may also own and post through multiple accounts. In this sense, Reddit is a pseudo-anonymous space that allows for users to build their own reputations while maintaining multiple, unconnected accounts (Donath 1999; Massanari 2013, p. 5). This feature acts as a shield for reporting possible cases of sexual violence: through the use of a pseudonym, users achieve what Nissenbaum describes as unreachability (1999). Users who choose to reveal personal information are protected from potential harms that arise from being identified and find safe means of reporting through this pseudo-anonymity. Some users choose to push the boundaries of this anonymity even further through the use of "throwaway accounts".

Throwaway accounts, and Reddit more generally, have many inherent safeguards to manage context collapse. Social network sites have been described by Marwick and boyd as flattening various audiences into one (2011). "People attempting to communicate with groups of people that are not normally brought together, such as family and colleagues, can find this challenging" (van der Nagel 2013, n.p.). Reddit's lack of identifying features associated with users means that Redditors are able to navigate context collapse with minimal difficulty. Even so, some users choose to disclose their Reddit username to family and friends. Here, the flexibility for account creation on Reddit allows users to compartmentalize. They can go beyond a pseudo-anonymous identity and use a throwaway account. The use of these accounts seems to be respected and supported 
amongst the community. In fact, challenging Reddit's reputation as misogynistic and hostile, users have actively protected the identity of OPs who juggle multiple accounts and online identities so that they may remain anonymous and safe.

Redditor A: 5 points 11 months ago

is this your throwaway?

OP: 2 points 11 months ago

Yes, why?

Redditor A: 5 points 11 months ago

i think your account switched back, all good

OP: 8 points 11 months ago

Yeah haha for a second I'd accidentally replied with my normal account that I have family on, thanks for not being a dick about it and giving the name away.

The expected, conventional use of Reddit involves a stable account, one that "records and displays a history of what the use has posted, voted on, or commented around the site" (van der Nagel 2013, n.p.). Donath (1999) refers to this history as contextual information about the user, and helps in curating their reputation online. This technological code can be subverted or challenged when users create a new, temporary account, referred to as a throwaway account, in order to hide contextual information and identity (van der Nagel 2013). In doing so, a user does not benefit from social voting in the same way. While an upvote or downvote may provide an OP with a sense of validation, karma points are not afforded to their primary account. Effectively, the incentive to only post content that will be well received by the community, and to pre-emptively screen for problematic or contentious content is gone: users have opted out of the "game" and goals of Reddit's participatory culture (Massanari 2013). 
van der Nagel's analysis of Reddit's r/gonewild subcommunity challenges the often riskaverse perception of online anonymity practices by analyzing the practice of users posting their own intimate images online. Despite the fact that online anonymity carries risk of abuse, harassment, fraud or other illegal activities, anonymity provides many social benefits (Marx 1999; Kling et al. 1999). Although anonymity removes accountability, it makes much needed space for freedom. Users can speak freely, without risk of surveillance or judgement on the basis of age, gender, status, appearance, or race (Donath 1999). An OP's choice to use a throwaway account to host these discussions speaks both to a perceived need to protect their identity, but also to authenticity. An OP using a throwaway account commented on the challenges he had received to his narrative. Many assumed that he had fabricated parts of his story to convey sexual activity that happened outside of a monogamous relationship as assaultive; he was accused of lying in part to avoid accountability for cheating on his wife. In response, OP clarified the choice and intention of posting on Reddit. His comment reinforces that the nature of these posts is genuine and those who post are grappling with complicated and nebulous understandings of their relationship to others, sexual boundaries, and the law.

OP: 1 point 3 years ago

I'm just going to put this little diddy right here. I value your opinion, if $i$ didn't want it I wouldn't have requested it. I don't expect you to believe me, nor do I expect to be completely absolved from what has transpired. I would just like to point out that "reddit' isn't going to make what transpired go away. Nothing anyone says or does in this forum is going to change what happened or what will happen. I have nothing to gain by posting this here, nobody is going to "defend" me to my wife about what transpired so what exactly would be my end game for lying ...on reddit? 
By rhetorically asking what his "end game" for lying would be, OP suggests that this is a space where, to truly glean a benefit, one must be authentic. In understanding human interactions, specifically impression management, many refer back to Erving Goffman's work on dramaturgy (1959). Here, OP is clear: there is little need for impression management, and Reddit has little to offer him outside of an opinion. By OP's characterization, his audience - specifically, their opinion of him - does not matter. The front stage performance that Goffman suggests governs our day to day societal interactions is temporarily suspended or, at the very least, clouded. I would suggest that in many of these discussions, the poster is able "to relax, he can drop his front, forgo speaking in lines, and step out of character" (Goffman 1959, p. 488). While it is impossible to say that this is the backstage of Goffman's theory of dramaturgy, I believe this is as close as research may get to discussing such politically charged and socially fraught topics with complete authenticity. ${ }^{11}$ Conducting an analysis of these spaces provides a lens to engage in a deep, nuanced critique of the grey areas of both sexual boundaries and law, and a respect for those who find themselves (or their experiences) toeing the line between acceptable and assaultive sex.

My reading and analysis of these discussions has been informed by Janet Halley's suggestion to "take a break from feminism," allowing my work to explore potentially

\footnotetext{
${ }^{11}$ In saying this, I recognize that near every form of social life is performative and relational. The argument I am making here is not that we are seeing a completely authentic 'back stage,' but rather, a closer, more authentic representation than might otherwise be visible. Anonymity does not entirely remove personal motivations to lie or misrepresent, but data like this is likely to curtail a great deal of impression management and intentional misrepresentations.
} 
unseen readings of grey-rape, or ambiguous sexual interactions to move beyond a feminist politic of injury (Halley 2006; Khan 2014c, p. 261). This "break" intends to shatter what many have accepted as a dominant framework for sexual assault under the societal climate of rape culture. In taking a break, I also do not accept as fact that sexual assault is a gendered crime: a great deal of scholarly work on rape promulgates this on the basis that rape is a crime predominantly victimizing women committed overwhelmingly by men (Plaxton 2015; Gotell 2012; Bourke 2007; Brownmiller 1975). To accept that sexual assault is gendered would permit me to infer that OPs who asked if they were victimized were women and those who asked if they had committed an assault were men. However, in my analysis there was a substantial number of men asking about whether female intimate partners had assaulted them. There were even more posts where OPs chose not to identify their gender, nor made any reference to anatomy to situate their sex. Rather than commit an erasure of gender-queer, nonbinary, trans-identified, or male identity within this population, my work decentres gender and sex from its inquiry.

\section{$\underline{\text { Conclusion }}$}

To demonstrate the presence of a virtual judging community, my project relies on an ethnographic content analysis (ECA) of Reddit. Although Reddit has received little academic treatment, it is an exceptionally well-suited environment to conduct research on the boundaries of permissible and socially acceptable sexual conduct. A large part of this is due to how the site constructs and makes space for user anonymity. Past scholarship has described Reddit and Reddit's user base according to a narrow, restricted framework that is unable to account for the care and respect that users have afforded to these 
discussions and to the OPs who navigate these contentious and deeply personal questions. Reddit has evolved beyond a link-aggregation site into a diverse ever-expanding subset of distinct communities. In order to challenge the popular conception of Redditors as messy, karma-collecting misogynists, my project examines the spaces that are made for sensitivity, compassion, and nuanced discussions of sex, kink and violence. In suspending the widespread academic conflation of sexual assault and gender-based violence, my work aims to connect more deeply with the experiences and sentiments held by those who have been subjected to (or who have perpetrated) sexual violence. It also aims to unpack the tensions caused by the promulgation of a one-size-fits all consent framework that is often unable to account for the nuances of consent in practice. 


\section{Chapter 4: Findings and Analysis}

The findings presented were derived from a deep reading and analysis of 66 original posts on Reddit, which included over 4,000 comments. Findings have been grouped into broad conceptual claims that are concerned both with the rules and patterns of judging emerging from subcommunities as well as the nature of both subjects and subcommunities. The intention is not to guess at or reveal a user's original intent (conscious or otherwise) behind posts or comments. Rather, the claims presented emerged from observing the interactive exchange between posts and comments, OPs and Redditors, widespread community (dis)approval, and present or overlapping codes. The focus here is on both original posts and comment discussion as operational and a lens through which to view public perception.

\section{Limitations}

Before moving to the analysis and subsequent findings, it is important to outline some limitations to this project. Perhaps most notable is the limited scope of my inquiry and online data collection. Virtual trials take place in a variety of forums and online space outside of Reddit, and were also observed on other subreddits that ultimately were not collected and analysed. The decision to exclude data outside of five identified subreddits was made due to inherent time constraints in the research process, and also to ensure a focused analysis that explored a wide range of sexual practices and interpersonal relationships. By only having access to textual data, my research is limited as communicative nuances that would be more apparent through other research 
methodologies are obscured. A final limitation came from my decision not to follow up with subjects: I chose not to elicit feedback from the community; this means there is a limited ability to establish demographic information as I am wholly reliant on what is included by the OP at the time of posting. Although the scope of my inquiry was restricted and limited to text, images, and links, Reddit still provided a wealth of data critically engaging with questions of consent, sexual exchange, and assault.

\section{Consent as Gatekeeping}

A common theme that emerged throughout the analysis was the pervasive understanding of consent as a form of gatekeeping. Gatekeeping is understood as one party granting permission and access to their body after being petitioned by the other party. Past work has grappled with these narratives or 'sexual scripts' and explored what dominant consent frameworks can tell us about sexual conduct as it situates itself in ever-changing historical and cultural environments (Simon and Gagnon 1986, 2003). Jaclyn Friedman is credited with popularizing recognition of the 'gatekeeper model' of consent, where women's sexuality is commodified and women are socialized to 'gatekeep,' or manage access to their bodies through granting and denying consent (Gunsaullus 2015). Below, an OP's post exemplifies this model in action as she questions whether a failure to prevent an assault, and her choice to eventually "[give] in" meant that she granted consent.

$O P:$

I'm just trying to get this straight in my head. I don't know if I was raped or not on Saturday night and I would really like your honest view. I'm not trying to be dramatic but 
it really upset me the next day and I just want to know if it actually was rape or if I'm the one to blame here.

On Saturday night, I went on a first date with a guy. Dinner went great although we were out 'til really late as my car broke down on the way to the date and we didn't end up going out until 10:30. Because of my car, I met him at the train station near his house and he said he would drive us there. We had dinner and finished the date at about 1pm. He asked if I wanted to go back to his place and keep talking for a bit and I told him, I'd rather go home as it was late. He begged me to keep talking for a little longer and promised that he wasn't going to do anything "untoward". He was going to drive me home and I had no other way of getting there as the trains stop that late back to my house. So I reluctantly (and stupidly) said ok then.

We talked for a bit longer and we made out a little (which I was ok with) but then he started trying to put his hands under my clothes. I kept pushing his hands away and saying that I thought we should get to know each other better first. He kept saying "it's ok, I promise I won't do anything". I kept pushing his hands away and he'd slow down for a minute or so and then do it again. I kept saying stop I was physically pushing him and he was resisting me when I pushed. At one point I said "stop, get off me!" And finally he did. I was freaking out and I kept saying "please, I just want to go home" he apologised and said he didn't mean to make me feel uncomfortable and just give it five mins and he would take me home. I calmed down a bit. He had told me he hadn't been with a woman in about a year and I somehow rationalized this behaviour as he was just really excited as it had been a while. He apologized again and I said. It was ok. (I so regret not just walking out and getting a taxi, I know I'm an idiot and I should have taken more steps to get out of the situation)

He started kissing me again and although I was uncomfortable I thought he'd keep it to kissing as he had apologised and realized he had upset me. In all honestly, I don't know why I let him kiss me again. I should have just left. I am so ashamed of myselffor being so stupid.

He started putting his hands under my clothes again and I kept pushing him away again, saying I wanted to wait. He kept saying he wasn't going to do anything. At one point I thought "the only way I'm going to get home tonight is if I just do it" so I stopped saying no and just had sex with him. He was really rough and I'm covered in bruises. It didn't hurt because I had stopped resisting though.

I know there are so many more things I should have done to prevent it, starting with not going upstairs and then just leaving after the first part but I'm so upset. Was this rape or because I gave in eventually was this consensual? I don't really want to report it but I just want to know one way or the other so I don't feel like I'm crazy and making it up or so I just suck it up and get over it.

tl;dr: kept saying no but eventually gave in. Was I raped? 
Neoliberalism has been said to enjoin citizens to adopt self-governance, accompanied by an excessive focus on individual responsibility and risk management (Hartman 2005, p. 63; O'Connor, Orloff and Shaver 1999). In this light, gendered differences are less visible and cultural logics that blame women who experience sexual violence may be amplified and supported by placing the responsibility on their failure to manage their own risk (Salter and Crofts 2015). In order to effectively be able to manage risk in sexual encounters, a neoliberal position would necessarily view romantic relations as a deliberative negotiation, not unlike a contract (Salter and Crofts 2015). Under the logic of a contract, gendered power imbalances are naturalized along with social and historical structures that may complicate or contextualize sexual interactions. Gatekeeping is reinforced as a logic and upheld, where OP feels she must acknowledge some form of responsibility for the violence perpetrated against her. Further, this contractual understanding "overlooks the spontaneous and embodied dimensions of sexual practice that do not lend themselves to calculative rationality" (Salter and Crofts 2015, p. 3).

Despite OP carrying blame, the comments were supporting, affirming, and validating. The community universally assessed this script as assaultive. In the comments, only one Redditor reinforced the OP's original victim-blaming narrative, writing “you are partially at fault here... you were clearly uncomfortable, but you stayed. You said nothing about being drugged, you said nothing about him forcing you down and pinned". This user invokes a calculative rationality where OP would be expected to fight back and prevent her assault from taking place. By arguing that OP possessed the means to stop an assault, 
responsibilities are blurred and a gatekeeping model is further supported. Her staying is presented as a choice and more weight is placed on the victim to appropriately navigate sexual scripts and uphold a defensible position.

Throughout the course of my analysis, it became clear that the gatekeeping model was a near universally accepted sexual script. While it was much more common to read posts and subsequent discussions like the one above, there were also several posts where users (often identifying as men) asked about their involvement as possible perpetrators of sexual violence. A gatekeeping model of consent was mobilized similarly to place responsibility on the victim and offset any blame that could be directed at the OP.

$O P:$

Reddit here is my story that might not be appropriate for this subreddit but here goes nothing

A few years ago I was dating this girl. Let's call her Alice. Alright Alice and I had been dating pretty much all of high school and everything was dandy. We lost our virginities to each other and all that cute high school couple bullshit So one day junior year(this is 5-6 years ago) I remember I had my house open. Being the horny teenager I was, I had her come over.

A little something that might count, she bought a few thongs a couple days before this and being the horny teenager I was, my dick was already out of my pants.

Anyway, so we end up Netflix and chilling. I egg her on for sex cause she has these new thongs on and she denies me. I get disappointed and basically beg her (pathetic, I know). All of this is hazy, so every detail may not be there. At this point I'm trying every trick in my teenage book. "Just the tip?"

So from what I can remember I get a silent okay. Which to me now, sounds like I was getting mixed signals. I put the thang in for about a minute and notice her not giving me any emotion. I stop. I don't finish. I just stop. After that it's even hazier. I don't remember shit after that.

However, I do remember a couple days later she claims that I raped her. Just so you know, it might have been teenage love but goddamn it I loved everything about this girl 
and I'm guessing she felt the same way. We told each other everything and I was pussy whipped. Now that I'm older, I realize maybe she really didn't love me back. With that being said, I would never knowingly rape someone that meant everything to me at the time. So I see her again at her house after she claims that I raped her. I'm beating myself up mentally and physically because I can't believe she said I did that to her. What's fucked up is we end up having some sort of make up sex right after this episode of me beating myself up.

Anyway fast forward to now. She still claims that I raped her. I'm going insane here Reddit. Constantly going back in forth in my mind. I loved this girl with everything I had and had no intentions of doing something to this magnitude. I'm the type of person that questions everything I did 17 minutes ago

Reddit I wouldn't lie about this. This is everything I remember. Did I rape my ex girlfriend?

Here, the OP's behaviour echoes many sexual scripts now being presented in the \#MeToo movement; in fact, it closely resembles Babe.net's piece on Aziz Ansari. ${ }^{12}$ However, the OP clearly attempts to establish an underlying power structure predicated on unrequited love. Rather than accept that his behaviour was pushy or aggressive, he presents himself as emotionally vulnerable, "pussy whipped". "[Loving] this girl with everything [he] had" builds a case that he could not have raped her. Sexual assault and love are thus seen as polarizing, or at least, love is a mitigating factor. This does little to change the opinions in the comments, and the community overwhelmingly takes the position that OP raped his ex-girlfriend. The top comment establishes the majority opinion, but is empathetic towards the OP, placing the blame instead in an "evil misogynistic culture."

Redditor: 11 points 8 months ago

Yes, sadly you did.

\footnotetext{
${ }^{12}$ See https://babe.net/2018/01/13/aziz-ansari-28355. "Grace" details a grey rape encounter with Ansari following a date. Similar to the OP, Ansari continued to escalate sexual advances despite initially receiving signs of non-consent.
} 
She did not want to have sex with you and told you so but you persisted despite her telling you no, and she evidently felt so pressured that she decided it would be 'easier' to let you enter her body than not. Your prior or subsequent interactions with her have no bearing on the single incident you have described, neither does the question of intent. She did not consent. A 'silent okay' is not consent. You committed rape. And I think deep down you are beginning to understand this, which is why you are posting on this sub.

Now that's clear, I want to add that you should speak to a therapist about this incident and how you feel about it. It is important that you accept personal responsibility for what you did, that you understand the reasons why (including the rape culture that encourages young men to believe such attitudes and approaches to sex are acceptable) and that you discuss it in depth with a professional for the sake of your own mental health. I have no suggestions as to how to handle it with your former partner if you are still in contact. I believe rape survivors may best advise you on that.

Finally, no one person can be defined by a single act. I hope that the damage this evil misogynistic culture has done to you can be rectified by your acceptance of your own failure here, and if you ever father a son or a daughter, make sure he or she knows that only yes means yes.

Despite the fact that a "silent okay" is dismissed as inadequate consent, comments still invoke logics inherent in the gatekeeping model; lawful consent hinges on a yes from the responding party and, should they be coerced into giving it, the "yes" has no power and the gate remains firmly shut. Establishing consent as a unilateral process, where one party remains wholly in control of granting and the other responsible for reading implicitly suggests a feminine quality underlying sexual violence (see Du Toit 2009). Women have historically been imagined as passive participants in sex, whereas men are imagined as sexual aggressors and primary actors (Du Toit 2009). The responses received by OP demonstrate that the community as a whole places consent granting in her hands and dismisses inaction as a form of consent. Even after acknowledging the cultural failings to move beyond a patriarchal framework and understanding of sex, where women are passive and men are aggressive, the comments reinforce that it is the dominant model with which to understand consent and, consequently, sexual assault. 
OP: 3 points 8 months ago

Why wouldn't she tell me to stop immediately? I obviously feel horrible about this. I haven't been in contact with her for years. It's just come up again. I can't live with myself right now. I'm that fuckin freak, you know? I never intended that.

>Redditor: 10 points 8 months ago

You don't take her no seriously before, why would you take it seriously after she repeated herself again?

>Redditor: 9 points 8 months ago

Well, it sounds like she kept saying she didn't want to, but you kept pushing her for sex?

>Redditor: 6 points 8 months ago

If you've been pressuring her, and she's having sex against her will, and she's incredibly vulnerable, it would be incredibly hard to find your voice (which has previously been ignored) to tell you to stop.

You may not have intended to rape her, but you have done. If you're struggling with this (and by all rights you should be), it would unfortunately be very difficult for you to seek help from a therapist. I understand that mandatory reporting only covers minors/elders/dependent adults, but since you appear to have committed the act before you were both 18 I'm not sure what would happen.

>Redditor: 7 points 8 months ago

She told you to stop multiple times. How many more "no"s did you want?

The gatekeeper model is premised on the idea that our cultural scripts surrounding sex and consent today originated from our history. Sex is unilaterally sought out by one person, and safeguarded by another. This hearkens back to traditional marriage arrangements where men would provide financial security in exchange for unrestrained sexual access to their wives, governed further through the legal imagining of women as 
property who could not be raped by their husbands. By feminizing resistance to rape and imagining women as passive sexual participants, a gatekeeping framework for consent continues to put forth outdated assumptions about women's sexuality. They are predominantly understood and assumed to be gatekeepers. A great deal of the Reddit posts implicitly supported this understanding. While I had about a dozen male-identified OPs who questioned whether they had been assaulted (and many more who did not specify their gender), a violation was often non-negotiative. Women who perpetrated sexual violence rarely sought consent or attempted to persuade partners: instead, men were often victimized while intoxicated or sleeping, or an assault occurred through a unilateral and deceptive choice to not use a barrier device. Deceptively navigating condom use was one of the most common ways that men felt raped. One such scenario happened to follow in a similar vein to gatekeeping, where the OP's consent was solicited again and again under threats and coercion until he was worn down.

$O P$ :

This could contain some triggers, I'd think. Be cautious!

So, I'm not upset or bothered by this, but immediately after this happened I was super paranoid and pissed off.

About 2 to 2-1/2 years ago, I met a girl on Tinder. We went to dinner, had sex, and started casually hooking up a few times a week. I was 21 and she was 19 or 20.

Fast forward a couple months, we still hook up occasionally, and we both agree that we aren't being exclusive or anything serious beyond hooking up(she seemed reluctant but agreed, and I was VERY clear I was seeing/hooking up with other women and not going to be in a relationship with her.)

Anyway, the last time(I believe) we had sex. We were at her place. I was always VERY careful and serious about using condoms with everyone. Never had sex without one. This time, she was really, really wanting me not to. She swore up and down that she was on birth control and was clean, yada yada. I continued to tell her no. She finally agrees that 
we don't have to and we have our normal, consensual, mediocre sex. After I finish, we're laying there in the dark and I let her know I'll be leaving in about a half hour.

She begins to beg for sex without a condom again. I tell her no, being nice at first, but she is very persistent. She starts getting really upset and angry, but I continue to say no. I get up to leave, and she yells at me. She yells, "Wow! Really? You're going to leave now? Are you sure that's a good idea?" I don't know why, but I got scared and started to think she might file a false rape report if I left. I know it sounds like I was jumping to a huge conclusion, but she was really scaring me and I just had a feeling.

I stayed to talk, trying to defuse the situation. It wasn't happening. I kept saying no, and she kept begging. She pulled me onto her and was rubbing on me until I was semi-hard again. She started to pull my penis closer to her vagina, and I pulled away. She got really pissed again.

I sat next to her while she laid on the bed and we argued about it again. I was scared, so I secretly pulled out my phone to record the conversation to cover myself. I made sure to be clear and say things like, "Are you serious right now? You know I don't want to have sex without a condom. And you're essentially forcing me to? What if I don't? What do you mean I'll regret it?"

This continued, I got scared, and we had sex for like 4 pumps without a condom until I acted like I was cumming and pulled out. She was satisfied and all happy about it after. I was still bummed but acted happy. I ended up leaving like a half hour later.

I listened to the recording on the way home, and you could hear absolutely nothing. It was useless.

There are small differences in this narrative from the female OP's earlier. Despite the fact that this OP also stays and complies, he does not engage in self-blame. In the comments, unlike the female OP, he has no intention of reporting the incident, and yet he still takes steps to collect evidence. This may be connected to the continued history of belittling and dismissing male victims of sexual violence (Abdullah-Khan 2008). However, this post suggests that, in practice, a gatekeeping model may transcend and even subvert its theoretical underpinnings. Seeing such a stark role reversal with a model that is intended to highlight the gendered elements of sexual violence demonstrates that: a gatekeeping model of consent may exist irrespective of power dynamics, and that research on sexual 
violence has a great deal to gain from challenging pervasive frameworks and suspending its traditionally gendered inquiry.

\section{$\underline{\text { Risk Aversion and Post-Sex Anxieties }}$}

As discussed earlier, rape is often imagined in both law and society as the ultimate violation of one's body. In the law, it is treated as an exceptional crime, and those who are convicted are subject to more severe maximum penalties that perpetrators of nonsexual assaults. This suggests that "sexual harms are viewed as more traumatizing and qualitatively different than other types of harms, because of their putative political, symbolic, psychic or physiological effects" (Khan 2016a, p. 1). In this sense, sex is seen as exceptional, as foundationally different from other offenses, and victims of sexual offending carry this social understanding with them. It has been argued that when rape is conceptualized in this way, as the worst form of violence that changes its victims forever, the message to victims is that they will never recover (Halley 2006).

There is an anxiety surrounding bad sex, grey rape, and ambiguous consent because of both the risk of criminal sanctions to an accused and the assumed lifelong psychological damage faced by the victim. As discussed earlier, rape can be imagined as a new site of sexual regulation and a means of becoming and unbecoming citizens. In these posts, many OPs sought to interrogate past experiences that they had previously left unquestioned, looking to disrupt or reframe a past narrative. In the cases of OPs who 
worried about being victimized, there was a distinct privileging of harm and trauma, where grey experiences were seen as needing definition and closure.

$O P$ :

I just realized a few months ago that I hooked up with a girl, and the day after I asked her how drunk she was last night, and she said she didnt drink at all. But I was pretty drunk. We only got to 3rd base, but was I raped?

Nearly every question is posed using the term rape. Despite the fact that rape is established through penetrative sex, users seem attached to this term and resistant to Redditors who attempted to label the experience as "sexual assault". This attachment appears to be connected to the psychological and social gravity of rape; the trauma that it affords its victims may help them to feel validated or to have what they believe is a violation taken seriously. Rape is mobilized across these platforms, alongside social discourse, as the pervasive symbol to represent all sexual harms (Khan 2016b). Perhaps the most exceptional use of the word "rape" was when one OP used it to describe kissing.

$O P$ :

I'm sorry if this isn't the right subreddit, but I mainly just need advice, as I'm not even sure that I was actually raped.

I don't remember exactly how young I was, but I do remember my brother for a period of time would call me into his room to 'practice kiss' me. I would lay down on his bed and he would lie on top of me and kiss me. He was/is 6 years older than me, and while the memories are foggy, I remember I really, really didn't like it.

I'm 20 now, and I only started thinking about this on my drive home yesterday. I don't think I really forgot it or repressed it, but the more I thought about it yesterday, the more I realized how wrong it had actually been. The thing is, I never once said no, and I don't think I ever put up any resistance. 
My brother and I are friends now. We have a good relationship. Neither one of us has ever acknowledged it since it happened. I don't know what I should do, or if I should even tell anyone since I'm obviously over it. I really don't even know how I should feel about this. Thoughts?

Here, OP is grappling with something that happened in the formative years of his life, that he acknowledges has no bearing on his life now or his relationship with his brother. In the comments section, Redditors are quick to ask him for clarifying information on his age, and attempt to distinguish criminal harm from experimentation (e.g. one Redditor noted that " a 10 year old 'practice kissing' his 4 year old [brother] is very different to a 15 year old doing the same to a 9 year old"). While many suggest that this behaviour is better understood as molestation, the majority suggest that OP seek professional help to move on rather than confronting his brother or dwelling on the event. The OP does not re-engage in the comments or provide any contextual information, but would find little guidance in how to make sense of this experience as he received wide-ranging and contentious comments. In many of these posts that seem prompted by underlying anxieties and sexual exceptionalism, OPs are missing key details necessary for a criminal investigation or thought experiment. In not providing an age framework, Redditors struggled to hold the OP's brother accountable. Similarly, one OP was unable to account for her experience altogether due to intoxication, but desperately wanted to give it a label.

$O P$ :

I'll cut to the chase. My boyfriend and I have a highly active sex life, but we aren't always able to do the deed all the time because if family and friends.

Recently we were at a party and got completely drunk. We took an Uber back to my parents place and were still pretty smashed. 
It's foggy but I'm pretty sure we had sex that night, but to be honest I don't remember clearly and I never said yes to it. We woke up on the same bed together.

Was it uncool of him to do that? I never consented and now our whole relationship feels weird to me in the back of my head.

OP titled this post "Was I raped? [Rape]" and, despite asking in her post whether the behaviour was "uncool," what she is really interested in is whether it might be assaultive. Here, despite not knowing whether or not she and her boyfriend had sex, the OP instead focuses on how she could not have provided consent if they did. She acknowledges that they have an active sex life and both had a lot to drink, but rather than allow for grey areas in their relationship, or extending to her boyfriend a mutual inability to provide consent, she re-enters the comments occasionally to remind Redditors that consent would be impossible for her. The comments here focus on the messiness in making this accusation and direct OP to resources such as therapists and RAINN.org. Interestingly, very few comments suggest that OP have this conversation with her boyfriend, which continues to stress the legitimacy of spaces like Reddit and other institutionally based authorities as providing the ultimate iteration of consent.

Placing the power to dictate what consent looks like in institutions means that "good sex" is often productive sex: otherwise put, monogamous, marital, in-the-home, in-love, etc. (Khan 2016a). There is little space here for sex "for the sake of pragmatics or pleasure," and sex that does not serve a societal purpose or sex that is seen as inherently risky is viewed as problematic and even justifiably criminalized (Khan 2016a, p. 1). It is under this environment that certain spaces can be used as resistance or counter-culture 
education. The posts discussed so far in this chapter have all surfaced from r/sex, $\mathrm{r} /$ relationships, and r/relationship advice, however there seems to be a uniquely productive and purposeful engagement with these posts on $\mathrm{r} / \mathrm{bdsm}$ and r/BDSMcommunity.

\section{BDSM Communities as Educators}

While BDSM affiliated subreddits also tend to imagine consent as a gatekeeping process and experience similar post-sex anxieties and risk-averse views, the comments that surface in these threads are qualitatively different from the other three subreddits. Redditors are less prescriptive when addressing the fact scenarios within posts and give more deference to education and to how the OP feels. In this space, there also seems to be an underlying acknowledgement that visitors may not align or identify themselves as members of a broader BDSM community. $\mathrm{r} / \mathrm{bdsm}$ and r/BDSMcommunity have well over 200,000 subscribed 'kinksters'. Each subreddit, unlike the other spaces analyzed, is quick to welcome Redditors in the side panels normally reserved for community descriptions, rules, guidelines, or links to other resources. Unlike every space analyzed, both r/bdsm and r/BDSMcommunity present clear and organized rules that seem to focus on inclusivity. The images below show the subreddit identification panels and the community rules and guidelines for both BDSM affiliated subreddits, as well as r/sex to serve as a comparison. Whereas $\mathrm{r} / \mathrm{sex}$ involves their community of Redditors in policing fellow Redditors' use of space, r/bdsm and r/BDSMcommunity seem more focused on a user's ability to self-govern their behaviour in a minimally restrictive fashion. 


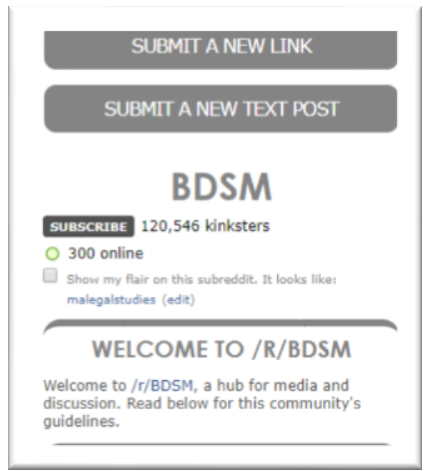

Image 1: Banner on $\mathrm{r} / \mathrm{bdsm}$

SUBMIT A NEW TEXT POST

BDSMCOMMUNITY

SUBSCRIBE 90,401 kinksters

O 447 online

Show my flair on this subreddic, It looks like: malegalstudies (edit)

WELCOME TO BDSMCOMMUNITY

A hub for discussion, questions, help and
conversation, Read below for this community's guidelines.

Image 2: Banner on r/BDSMcommunity

Submit a new text post

sex

910,580 readers

7,628 users here now

/ sex is for civil discussions about all facets of sexuality and sexual relationships. It is a iex-positive community and a safe space
or people of all genders and orientations.

ILEASE READ the FAQ with the most asked and

inswered questionsi before posting!! Posts that fo not follow the posting guidelines in the

IAQ will be automatically removed.

गLEASE REPORT ANY INFRACTIONS BY ZUCKING THE "REPORT" BUTTON UNDER THE POST OR COMMENT

thasee rasat!

Image 3: Banner on r/sex 


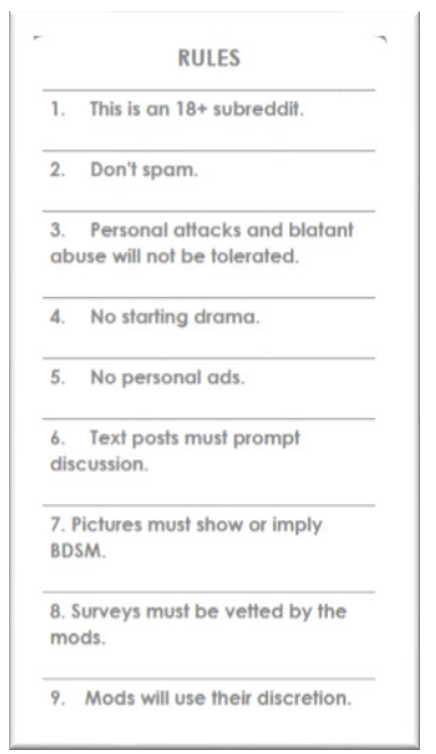

Image 4: Rules from $\mathrm{r} / \mathrm{bdsm}$

\begin{tabular}{|l|}
\hline \multicolumn{1}{|c}{ RULES } \\
\hline 1. $\quad$ This is an $18+$ subreddit. \\
\hline 2. $\quad$ Don't spam. \\
\hline 3. $\quad$ Personal attacks and blatant \\
abuse will not be tolerated. \\
\hline 4. $\quad$ No starting drama. \\
\hline 5. $\quad$ No personal ads. \\
\hline 6. $\quad$ Posts must prompt discussion. \\
\hline 7. Surveys must be vetted by the \\
mods. \\
\hline 8. $\quad$ Mods will use their discretion. \\
\hline
\end{tabular}

Image 5: Rules from r/BDSMcommunity 


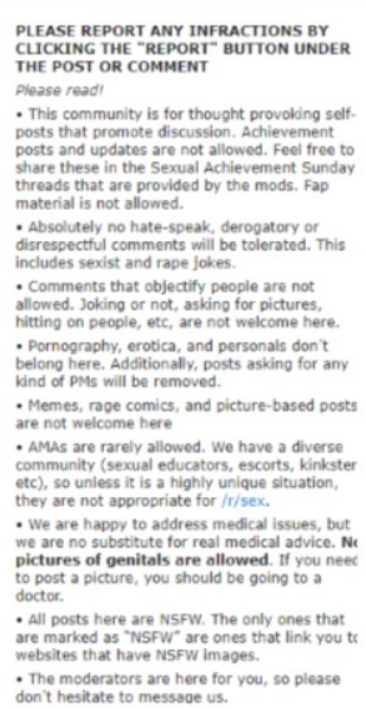

Image 6: Rules from $r / s e x$

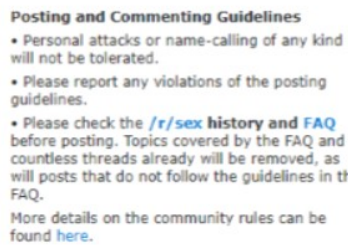

Image 7: Guidelines from $r / s e x$

An interesting theme that emerged from these subreddits was the communities' desire to spread knowledge. When analyzing the post in its entirety and coding for educating OP on consent, the average post from a BDSM affiliated subreddit had a coverage of $17.31 \%$. This means that nearly a fifth of every discussion analyzed focused on providing clarity in the form of education rather than an authoritative answer. Every single post to a BDSM affiliated subreddit received comments that were intended to educate. Sometimes this involved hyperlinks to educative illustrations, articles, or other online communities. Conversely, of the 55 posts that did not originate from a BDSM themed subreddit, only 
28 saw Redditors offering the OP any education; nearly half of the posts made in a subreddit that was not $\mathrm{r} / \mathrm{bdsm}$ or $\mathrm{r} / \mathrm{BDSMcommunity} \mathrm{received} \mathrm{no} \mathrm{comments} \mathrm{that} \mathrm{were}$ intended to educate.

The choice to educate an OP may come in place of providing an answer, or may be used to support their assessment and build a rationale as to whether the act was assaultive.

BDSM communities hold two relatively polarizing positions with regard to consent: the most popularly accepted model is 'safe, sane, and consensual,' whereas edgeplay practitioners often adopt a framework premised on risk-aware consensual kink (RACK). In my analysis of BDSM affiliated subreddits, while various forms of edgeplay were described, Redditors never acknowledged a RACK position or directly challenged others who advanced the 'safe, sane, and consensual' model. The absence of an overt RACK perspective may be due to tensions within BDSM communities and their representation in the mainstream. "Safe, sane, and consensual," particularly within the current culture surrounding consent, is likely to have more political traction within the mainstream (Khan 2014c, p. 259).

Redditor: 8 points 5 years ago

You mentioned being afraid to tell others about your experience because you thought they would think your BDSM lifestyle meant that you 'asked for it, or deserved it.' That is complete bullshit and anyone who thinks that way is ignorant and knows nothing about BDSM. BDSM is all about consent. It may be about control and power play, but both parties are consenting and enjoying it. Rape is one person getting off on violation while the other is truly destroyed. The difference between rape and a rape fantasy is that both parties in a rape fantasy love what's happening. There is no victim in BDSM. 
Redditor: 9 points 5 years ago

A key element that separates BDSM from actual torture or rape, is the consent of all participants. The moment consent of one of the participants is withdrawn, the scene should end immediately (hence the focus you see everywhere on the importance of a safe word). If the scene doesn't end straight away, the act moves from BDSM into rape.

That said, for people that expressly want to not consent, there is some wiggle room. Usually called consensual non-consent, it is always preceded by extensive negotiation wherein all parties express their desire and prior consent for this non-consent. Note that this doesn't apply to most D/s relationships, and as something that brings a lot of risk, should be treated with the appropriate care. Only if these conditions are met, which by your story it doesn't sound they had been, would I see a case where I wouldn't call it rape.

Comments continually framed BDSM alongside consensual pleasure. While many subscribed Redditors in these subcommunities may identify as kinksters, Reddit as a space blurs clear lines of belonging, and $\mathrm{r} / \mathrm{bdsm}$ and $\mathrm{r} / \mathrm{BDSMcommunity} \mathrm{are} \mathrm{likely} \mathrm{to} \mathrm{see}$ visitors who have more normative sexual inclinations. BDSM communities have been quick to critique their representation in the mainstream, from the dom/sub relationship in the popular erotic series Fifty Shades of Grey to Jian Ghomeshi throughout his highly publicized trial (James 2012a, 2012b, 2012c; R. v. Ghomeshi 2016; Grinberg 2014; Zanin 2014; Pfeuffer 2017; Sarah, Elliston and Blue 2015). In these spaces, they might shy away from voicing more contentious models of consent in favour of emphasizing the safety and consent that comes from pleasure.

Redditor: 0 points 10 months ago BDSM is all about SAFE, SANE, CONSENSUAL. You didn't feel safe, and it wasn't consensual. That is not BDSM. 
Redditor: 8 points 1 year ago

I would strongly urge you to also play by the Safe, Sane and Consensual motto. No drugs or drinking before or during play. If that is something you would like to continue, I'd make it a personal rule to only engage in those activities after you have gotten to know someone better.

RACK may be especially unpopular considering the tendency towards post-sex anxiety and risk aversion when choosing to host these discussions online. In many other subreddits, and occasionally even on BDSM affiliated subreddits, users seem to adopt a risk-averse stance to consent. Simply put, we can understand this stance as a 'risk-averse consent' (RAC) model that clashes against RACK. Widespread campus consent education stresses the need for consent that is enthusiastic, ongoing, verbal, sober, etc., which removes any agency to choose an acceptable or desirable risk when giving consent. Continually stressing that BDSM's ideology as a whole is "safe, sane, and consensual" is a means of establishing a more acceptable framework for consent and trying to present the community's standards as more in line with the mainstream.

A strong challenge to the framework put forward through J.A. in Canadian law and the denunciation of advanced consent is Halberstam's conceptualization of "queer time" as explored in the book In a Queer Time and Place (Halberstam 2005; Khan 2014c). Khan comments that this understanding can inform edgeplay as following "nonnormative logics and organizations of community, sexual identity, embodiment, and activity in space and time" (Khan 2014c, p. 258, citing Halberstam 2005, p. 6). Here, the hierarchical dyadic construction of risk and safety may be suspended or subverted; risk 
may be negated through the possibility of pleasure, or may even be exciting. One

Redditor commented on how time may be queered for members of the BDSM

community, and how pleasure may be felt outside of normative sexual chronology.

Redditor: 1 point 6 years ago

Just to contribute a little thought, not necessarily a critical thing, I have good friends who are heavy heavy into fear play. The sub does not enjoy anything about the play during it, but is immensely turned on thinking about it after. While this does not sound like the case here, there is to some extent a desire to push limits and experiment, because you don't know without trying whether you will like something, or even will enjoy remembering something even if you didn't like it until you've tried.

As everyone else has pretty much said, this sounds like it sucked/sucks and for that I'm sorry, but also it isn't rape, and in most relationships, perhaps more often in kink relationships we try something just to see and sometimes it works well, other times not, but talking about it and looking at it as a learning experience is the most productive way to move forward.

By advancing an alternative framework to understand pleasure, BDSM affiliated subreddits educate their community and challenge a risk-averse model of consent. These small challenges support theories like Halberstam's queer time and further intracommunity education as to acceptable challenges to vanilla risk-aversion. Seeing consensual sexual experimentation as a learning experience and, when not pleasurable, as bad sex rather than assaultive sex tests contemporary sexual ideology and heightened risk-aversion. In challenging the promulgation of a one-size-fits all consent framework, BDSM communities rely on other members to educate and make members aware of alternate means to assess consent. These challenges were neatly summed up by two users: 
Redditor: 1 point 3 years ago

In the beginning where boundaries are being set it would be important to get consent to clearly make sure with you partner what you plan to do. However, in a long-term relationship where you now know each other's kinks and what they like and what they have already consented to, it's a huge difference.

Redditor: 2 points 3 years ago

I think sometimes there's so much focus on verbal, explicit consent that we forget about moving into touch instead of away, mirroring, and other types of nonverbal consent. Consent communication has many paths, often using several types at the same time!

Despite posing some resistance, many in the community vie for mainstream acceptance. This is accomplished both through advancing the model of 'safe, sane, and consensual' over RACK, and through a clear vilification of dom(me)s who overstep, violate boundaries, or ignore safewords. This is reminiscent of the widespread condemnation of Jian Ghomeshi by community members. By self-identifying as a victim of sexual prejudice, Ghomeshi attempted to sidestep accusations and, perhaps disingenuously, present his behaviour as mutually consensual kink; after victims began coming forward contesting the mutuality of consent, the public quickly turned on Ghomeshi, and prompted discourse on dynamics of healthy BDSM relationships (Khan 2014a). Similarly, Redditors are quick to distance themselves and their community from "fake" dom(me)s, or individuals who capitalize on subs in the BDSM community to perpetrate abuse.

Redditor: 2 points 1 year ago 
People who genuinely are immoral and abusive, the type of person who would repeatedly rape, might very well find go into BDSM precisely because they find "Dom" to be an easy justification.

Redditor: 3 points 1 year ago

Putting on an act of scary or genuinely being scary in a mutually beneficial way is not the same as being an intentional rapist, which the above guy is. That said, any sub who would claim their dom ignoring their actual SAFEWORD is desirable, is not someone you ever want to play with. People who get into no-safeword RACK relationships don't do what that rapist does either, they've entered there though careful negotiation, and mutual trust. None of that from the rapist, he's preying on the ones he thinks he can get away with raping. Sadly not uncommon for newbies to attract scum like this.

Redditor: 9 points 1 year ago

jesus christ why is it that there are so many of these stories? Oh wait, I know. The predators who are too afraid to actually attack people use bdsm/being a "dom" to sexually assault people because they assume the survivor won't say anything to the police because they're worried about being outed as kinky. He violated your limits and your body and neither of those are what a dom would do. You had the unfortunate luck to run into a predator who took advantage of you and, not that it will make you feel any better, but I doubt you're the first.

Redditor: 7 points 8 months ago

He wasn't a Dominant. There's a hell of big difference between having some dominant personality traits and being a Dominant. If you're going to use the title of Dominant in a $D / s$ relationship, that means you get all of the responsibilities that go along with it. That means learning.

The clear identification and subsequent exclusion of fake dom(me)s is a means for the community to reinforce its values and protect its cultural capital and reputation. Despite this, Reddit becomes an accessible platform to introduce outsiders to the expected norms held within BDSM communities. Many users are directed to cross post (x-post) to a BDSM affiliated subreddit if it falls outside of the original subreddit's expertise. Here, 
the blurred lines on Reddit's subcommunities makes space for intracommunity education as well as further petitioning for BDSM's acceptance within the mainstream. Unlike other subreddits, they welcome new members and are minimally restrictive with community rules and guidelines. By advancing consent frameworks that align themselves with more risk-averse models, by categorically dismissing abusive dom(me)s as "fake," and by educating at a much higher rate than other subreddits, $\mathrm{r} / \mathrm{bdsm}$ and $\mathrm{r} /$ BDSMcommunity control their narrative. Education becomes a tool both to support struggling community members who may find themselves at odds with the law, and to vie for inclusion and acceptance within the mainstream.

\section{Male Reporting}

As discussed above, there was a surprisingly high number of male identified OPs using Reddit to navigate their experiences. Male victims of sexual violence get little attention in academic literature and, until recently, have been predominantly represented through studies of sexual violence in prison or considered within a homosexual subculture (Abdullah-Khan 2008; Javaid 2014). This reinforces a substantial body of literature that claims that rape and sexual assault is a form of gender-based violence perpetrated within a patriarchal culture. The use of safe, anonymized spaces by men to navigate these experiences challenges our tendency to conflate sexual assault with gender-based violence. Although demographic information was limited in this inquiry, if an OP identified as male or female within the body or title of their post, I made a note so that the use of Reddit to host virtual trials could be analyzed by gender. 


\begin{tabular}{|l|l|l|l|}
\hline & Victims & Perpetrators & Both \\
\hline Female & 37 & 0 & 0 \\
\hline Male & 12 & 7 & 1 \\
\hline Other & 9 & 0 & 0 \\
\hline
\end{tabular}

Although the majority of OPs identified as female and questioned whether they had been victimized, there were a substantial number of men who also questioned their status as victims of sexual violence. Circumstances surrounding these assaults were: not using a barrier device by force or coercion, or preventing use of the pull out method; intoxication or 'blacking out'; repressed memories; anal penetration without prior consent. Of these 11 possible assaults, 3 had been committed by another man, and the remaining 8 had been committed by women. Studies concerned with the prevalence of women perpetrating sexual violence are limited, and have only just begun to receive attention (Stemple, Flores and Meyer 2017). Attention towards female perpetration may be viewed critically, or as a means of upending a women's rights agenda focused on male perpetrated sexual violence; however, these examinations do not negate or minimize other forms of abuse (Stemple, Flores and Meyer 2017, p. 303). My focus here is to acknowledge the many forms that sexual violence may take, and to account for the diverse retellings of these experiences online. 
The experiences recounted by men near consistently involved some degree of incapacitation (through drugs, alcohol, or sleep), or a fear of being reported and not being believed. Despite a great deal of work describing the challenges men face when reporting sexual violence (see Javaid 2015; Stemple, Flores and Meyer 2017; Abdullah-Khan 2008), there were no qualitative differences in how male OPs presented their experiences. However, in many of these narratives, male OPs acknowledge that coming to terms with the possibility of being victimization took time and, occasionally, through being labelled as such by another person.

$O P$ :

Throwaway for obvious reasons. Backstory: This girl and I have been hooking up for a few weeks now, causal bordering on dating level. Anyway, her and I get drunk one night at a party, and I am drinking like its fucking Deep Impact and Morgan Freeman has told us we are all fucked. Anyway, lets call her 'Jenna' and I start making out. Totally normal. I wake up next to her the next morning, no memory of the night beyond the first party we attended and started to make out in. Jenna says that her and I had sex the night before and I don't think anything of it. So what I cant remember, I had sex, no problem.

Fast forward a week. Her and I are having buzzed sex, not to drunk but feeling good, but she seems out of it. Not like her. This chick is constantly hornier than a sailor in port, she gets wet at the drop of a hat, she is crazy. I ask her whats wrong.

She tells me last weekend when we were drinking like Stevo-O before 2008, her and I had gotten to my dorm room and I was considerably more fucked up than her. She says that $i$ 'flopped down on the bed, belt half undone.' Now this is when she already starts to get visible upset. She tells me that I wasn't exactly 'fully concious' and this is where she is vague and starts to trail off.

I kinda push her a little further and she tells me she got my pants off me and jerked me off until I was hard and rode me while I dipped in and out and she says she doesn't know how she feels about it and honestly I don't either. Any opinions? Please.

$O P$ : 
I was talking to the new girl and we got to the inevitable point about each other's past. I told her a story about this girl that I had sex with a few of times but we had little to nothing in common and it was just a fling. Well, the last time we had sex she was on top. We weren't using a condom, so when I told her to get off because I was about to come she locked her legs around mine and held my hands down. I came and it sucked being mad (kinda scared) and coming at the same time. Two opposite feelings, really. The new girl then said "You were raped." and I just sat there thinking about it. I never thought about it like that before, but it makes sense.

I'm not bitter about it anymore, and I lucked out because she didn't get pregnant (even though she claimed she did, but I made her take a test in front of me). But, I'm just curious. Was that technically rape? I do remember all the regret I felt that night and it didn't subside until I knew she wasn't pregnant. I was mad because she didn't do as I asked and did the exact opposite with force and I didn't want a child with her at all. So, it started consensual, but ended not so much. Also, I'm a not small, strong guy and could have easily have thrown her off me but, I didn't because the exact thing that went through my head was "shit, if I throw her, I might hurt her and then she might call the cops if she's this crazy."

For the most part, responses to these questions by Redditors is similar. They are not any more or less likely to identify behaviour as assaultive depending on gender. However, comments responding to male OPs will frequently petition the community as a whole to "reverse the genders," arguing that male victims of sexual violence deserve the same degree of care and respect. Conversely, across communities, Redditors offered male victims of sexual violence a consistent piece of advice:

Redditor: 2 points 3 years ago

Never stick your dick in crazy.

Redditor: 17 points 3 years ago

Dude, don't ever stick your dick in crazy.

Redditor: 4 points 3 years ago 
Don't stick your dick in crazy.

Redditor: 3 points 3 years ago

Moral of the story, don't be a dumbass and don't stick your dick in crazy.

Redditor: 3 points 3 years ago

You stuck your dick in crazy... what do we say about sticking our dicks in crazy? not today good sir, not today.

Men who reported sexual violence, not unlike women, received victim blaming from the community that responsibilized them to exercise care in sexual relationships. "Don't stick your dick in crazy" suggests an element of control that was not exercised by male victims and an instability in their female sexual partners. However, when not phrased as stand alone advice, this expression was almost always used in comments that were validating the experiences of male OPs. In this sense, “don't stick your dick in crazy” establishes culpability for female perpetrators while hearkening back to a similar ideology expressed through consent as gatekeeping: men are predominantly understood as aggressors in sexual encounters and women are understood as passive.

Redditor: 1 point 3 years ago

This has happened to me as well. Except I put up a fight and got the girl off of me before I tossed goo. Needless to say our fling ended there. Dont stick your dick in crazy, brother. Glad it turned out alright for you.

Male rape victims have a documented reluctance to report to police, and face neglect and a lack of support when accessing crises centres (Javaid 2015, 2016). Experiencing sexual violence runs counter to hegemonic masculinity, and past research has indicated that men feel dismissed or revictimized when seeking support through traditional justice systems 
(Javaid 2015). While conceptions of hegemonic masculinity are sometimes brought up by Redditors, these posts are always a minority opinion, and many other community members show support to the OP by downvoting dismissive or degrading comments. In this sense, Redditors facilitate safe(r) spaces for men to report sexual violence, or to feel validated when questioning their experiences.

\section{$\underline{\text { Conclusion }}$}

The analysis conducted on Reddit's virtual trials offers insights into a public conception of sexual violence across communities. When asked to engage with nuanced, deeply personal experiences, Redditors willingly and authoritatively grappled with the law and articulated legal judgements with little reservation. Redditors supported a conception of consent as gatekeeping, reinforcing ties to sociocultural history where women's sexuality was commodified. In doing so, we can see how the Reddit imaginary is in the process of evolving to a point where, irrespective of gender, men and women are expected to gatekeep and manage their own sexual risks. Discussions of consent have become increasingly risk-averse, and many OPs demonstrate substantial post-sex anxiety and discomfort with grey areas and subjectivity. Users feel pressure to label and describe their experiences to fit within uncompromising categories and, in posting online, communicate that they feel ill-equipped to make these judgements on their own. BDSM communities are especially conscientious in their engagement with these posts. They use their platform as a means of educating intracommunity members while aligning themselves with the mainstream tendency towards risk-aversion. Male OPs on Reddit may be seeking unique 
support outside of an authoritative decision, and are arguably more likely to find validation online than through traditional justice systems. While more popular technologically-mediated discussions of sexual violence (i.e. \#MeToo) are used to demonstrate solidarity and the prevalence of sexual violence, Reddit's engagement with more messy grey spaces of sexual violence flies under the radar. These discussions have yet to receive substantial academic or public attention; consent and sexual violence are continually imagined as black and white categories within the mainstream. This analysis challenges the certainty with which consent can be given or read, and points to institutionally taught frameworks of consent as a possible cause of anxiety and widespread moral panic. 


\section{Chapter 5: Discussion and Conclusion}

This chapter will further establish the sociocultural nature from which these discussions emerge. It will move to suggest future directions of research that may be considered through virtual judging communities. Finally, it will stress the importance of understanding these spaces and how OPs and Redditors grapple with grey or ambiguous encounters, often revising past sexual scripts to make meaning out of their lives.

\section{Consent as a Moral Panic}

At this point, this thesis has touched upon, but not yet fully unpacked, the current preoccupation in Canadian and Western culture with consent. This preoccupation, I would suggest, embodies or at least borders on a moral panic. The concept of a moral panic was first developed in the early 1970s by Jock Young and Stanley Cohen (Cohen 1972; Young 1971a; Young 1971b). Moral panics are typically understood as misplaced or exaggerated concerns of moral wrongdoing, resulting in persecuting innocent persons as well as the fear that certain individuals or groups might categorically threaten the wellbeing of society (Goode and Ben-Yehuda 2016). While I understand that there are critiques of this concept ${ }^{13}$, I use the term moral panic tentatively, and as an open

\footnotetext{
${ }^{13}$ The concept of a moral panic has migrated outside of academic work and has established itself within the public sphere. Scholars have suggested that this shift leads to methodological impurity and theoretical impiety (Miller 2016). Many still argue that moral panics have not been properly defined as a theoretical framework, leading to inconsistency and overuse (Best 2016). The label of a moral panic is assigned near exclusively to claims that scholars wish to debunk and expose rather than similarly specious claims that they support (Best 2016). This particular critique may explain why scholars have yet to consider contemporary consent culture as a moral panic and not simply a long overdue movement for women's rights. Indeed, Jock Young noted that the moral panic concept "would not, of course, be countenanced in respect of crimes such as rape" (Young 2009, p. 14). See Joel Best's "The Problems with Moral Panic" for further limitations of the moral panic concept (2016).
} 
invitation for others to question and critique the social response underlying contemporary consent culture.

Erich Goode and Nachman Ben-Yehuda were the first to identify the five key features of a moral panic: concern or initial social anxiety; hostility towards the perpetrators; a negative social reaction that achieves consensus; disproportionality ${ }^{14}$ with regard to the extent of the action or the threats it poses; and volatility with regard to media reporting and the associated panic (Goode and Ben-Yehuda 1994). David Garland later added two other features which he believed was more in keeping with Cohen's original intent: a moral dimension underlying the social reaction; and an understanding of the deviant behaviour as symptomatic (Garland 2008). I would suggest that all of these elements are present in the current social imagining of rape culture and heightened attention to issues of sexual violence and consent. Feminist discourses around rape culture have garnered a great deal of mainstream support. Rape is seen as an ever-present and pervasive threat, predominantly with focus on women as victims. The \#MeToo movement has engaged in widespread social vilification of accused perpetrators, and the public response to these 'outings' is reminiscent of public shamings and absent any due process protections and considerations. These stories are widely publicized and broadcasted throughout the media. Accusations of rape call question into the moral character of the accused and, within the climate of a rape culture, we assume that these behaviours speak both to the

\footnotetext{
${ }^{14}$ Disproportionality has received specific critique as a determining factor of moral panics. Arguably, interested parties who would speak to the media, report on issues, or write concerning topics may have personally experienced the phenomenon that gives rise to panic. In this instance, painting panic as irrational or disproportionate has been critiqued for ignoring the tendency for advocates of social problems to present these problems under an emotional rhetoric (Best 2016). However, when considered alongside a widespread social consensus and anxiety, these concerns are less pertinent.
} 
moral integrity of an accused while still being socially taught and ubiquitous. Consent is the dividing line between sexual experiences that are desired versus assaultive. The societal understanding that we live and exist within a rape culture draws our attention to this line, to behaviour that may push boundaries of what is accepted or desired, and to the importance of obtaining and articulating clear consent. I would suggest that consent is being institutionalized and mobilized in this way to build a barrier against the risks inherent in a rape culture.

Society appears subsumed with calling out and policing non-consensual behaviour- an admirable position - however, in these efforts and discourses, consent, and situations absent consent, are viewed with anxiety, distrust, and uncertainty. Judicial decisions have been elaborating and applying more rigorous standards of sexual consent; this consequently favours more normative sexual subjectivity that, at its core, is built upon anticipating, managing and minimizing sexual risks (Gotell 2012b, p. 252). The law is teaching us that consent is always universal, despite the nature of individual relationships, and always recognizable, despite the fact that miscommunication, hazy memories, and feelings of ambivalence are regular facets of life.

Miscommunications that occur outside of sexual scripts are unlikely to be criminally sanctioned. Outside of the law, miscommunications and even non-sexual violent assaults would not receive the same degree of public condemnation and shaming generated through the \#MeToo movement. This form of sexual exceptionalism has brought about the use of "consent captains" and the development of consent apps, as well as a focused 
educational effort towards younger and younger audiences (The Canadian Press 2018; Petter 2018; Knight 2018; Patel 2018). Most recently, Deanne Carson, a sexuality educator, recommended that parents ask consent of their babies before changing diapers (Patel 2018). The heightened governance and attention to consent has been building, and the transnational attention these issues are receiving are emblematic of a moral panic.

Consent has begun to develop an institutional script. While it makes sense for our law to establish frameworks to assess consent, consent is, and must continue to be, something that individuals assess by themselves. Using consent captains at bars suggests a separate form of institutional policing that takes agency away from individuals who would negotiate consent on their own terms (The Canadian Press 2018). Consent apps, such as Good2Go, We-Consent, uConsent, SaSie, and LegalFling, demonstrate a saturated market of litigious, risk-averse technology that captures wide-ranging sexual behaviours and subjects them to legal strictures.

The nature of these discussions on Reddit demonstrate that individuals place more trust in others to recognize consent than they do in themselves or their partners. This anxiety further reflects the social preoccupation with consent and with the dangers inherent in a rape culture. By asking third-parties to account for the very personal process of granting consent, these OPs reinforce that their consent does not belong to them and cannot be assessed individually. Testing consent against community standards demonstrates a loss, at least in part, of agency for those in sexually ambiguous situations and a need to share and defer this authority with a broader community. Some Redditors were quick to 
comment on and challenge this view, refusing to answer and instead commenting on how the OP framed their experience or the personal nature of consenting and their inability to judge.

Redditor: 29 points 3 years ago

You feel like you consented - then there was consent. Consent is up to you and you alone. No one else can state what is consensual than you.

Redditor: 12 points 3 years ago

Don't allow other people to rewrite your life for you.

Consent seems to have taken on an institutionalized master framework that individuals attempt to measure their experiences against. Making consent decisions is a necessary component of healthy, pleasurable sex, and as sex increasingly becomes the subject of public discourse and sensationalist media representations, the importance of understanding this process becomes evident (Cowling and Reynolds 2016). The current propensity to measure experiences against community approval and institutional safeguards demonstrates inherent challenges and anxieties surrounding sex. The law may see these challenges through complainants lacking confidence or surety, or through overbroad prosecutorial practices justified through risk-averse policy measures. People who have grey or ambiguous sexual experiences may find similar challenges in defining their experience or identity alongside consent and rape culture discourse. They may be confronted with labels, a societal pressure to "come out" as a survivor, or newfound epistemic privilege. By bypassing the criminal justice system, these Redditors suggest that an informal, anonymized assessment is their preferred first point of contact. This is unsurprising considering how labelling sexual experiences as rape debases both victims 
and the perpetrators. Perpetrators are subjected to carceral interventions, and victims are seen as fundamentally traumatized and forever changed by the experience; both are 'othered' within our risk-averse framework of sexual citizenship. These spaces on Reddit allow for a trial run of stories, where users may anonymously seek out a definitive narrative explanation of a personal event, how they should feel about it, and how others may accept or contend their status as a victim or perpetrator of sexual violence.

\section{$\underline{\text { Final Thoughts }}$}

This work is preliminary and the first inquiry into online spaces' engagement with the notion of consent and the construction of virtual trials. However, there are many questions left unanswered and many paths that may be taken in future research projects. Reddit is only one space of inquiry online, but also hosts other virtual trials outside of the identified subreddits and outside of the realm of sexual assault. r/legaladvice, for instance, is rife with users seeking community perspectives on legal issues and redress. Outside of Reddit, questions of sexual assault and violence are entertained in blogs, sexual education websites, and public and private forums. These spaces are all governed by different rules and guidelines for users. Indeed, a preliminary examination of feminist blogs that host virtual trials often place one user in a place of authority, not unlike a judge. The response gleaned is to be taken as the final answer, and users seem hesitant to challenge this structure. This project has also examined this process as the first inquiry into sexual violence, however these spaces may prove validating for victims who were unsuccessful through a criminal trial. Consent apps have only now moved to integrate responses within the blockchain; LegalFling in particular has moved to understand 
consent as a legally-binding contract that should be initiated prior to any sexual interactions (LegalFling.com). In the upcoming few years, we may see more drastic technological interventions and engagements with sex. These, along with the virtual trials examined in this project, are worthy of academic inquiry to trace conceptual changes underlying sex and consent.

Our experiences, and the stories we tell others about ourselves, give meaning to our lives. Humans have been described as a 'storytelling animal' (MacIntyre 1984). Our memories and stories are influenced by abstract ideas, moral judgements, more broadly, by societal frameworks (Tirosh 2017). The construction of memories is built through a 'sense of past,' but not the past itself (Confino 1997). In allowing a community to weigh in and provide meaning to an otherwise ambiguous sense of past, these users are allowing them to rewrite their stories, their narratives, and how they make sense of their lives. In revising and reinterpreting events under the guise of risk-aversion and anxiety, trauma is read into experiences that may better be left grey. For users who may have internalized rape myths however, these spaces may be liberatory and provide unique mentorship, guidance and community support.

These spaces, much like the experiences they judge, are grey. The OPs who remain active are often grateful to the community in helping them rescript these events. However, it is important to critically interrogate the results of these interventions. In seeking community support outside of traditional justice systems, law as a system of authority is destabilized. These users trust the community to make sense of their experiences through virtual 
judgement, and trust the community with an open, transparent accounting. Further examinations of these spaces may have much to offer research into sexual violence, technology, and the law. This project ultimately demonstrates the need for attention to these spaces, to alternate means and communities of judging, and to individual and institutional processes underlying consent decisions. At stake is an appreciation not only for unmet needs of victims of sexual violence through legal systems, but for how consent decisions are made, and how people navigate and understand these processes so necessary for an ethical, healthy, and pleasurable sexual life. 


\section{References}

\section{$\underline{\text { Legal Cases and Factums }}$}

Criminal Code, RS C 1985, c C-46, s. 271.

Pappajohn v The Queen [1980] 2 SCR 120.

$R v$ Al-Rawi, 2018 NSCA 10.

$R v$ Cey (1989), 48 CCC (3d) 480 (Sask CA).

$R v$ Creighton [1993] 3 SCR 3.

$R v$ Cuerrier [1998] 2 SCR 371.

$R v$ Daigle [1998] 1 SCR 1220.

$R$ c Daigle 127 CCC (3d) 130 (QC CA).

$R v$ Daviault [1994] 3 SCR 63.

$R v$ Esau [1997] 2 SCR 777.

$R v$ Ewanchuk [1999] 1 SCR 330.

$R v$ Ghomeshi, 2016 ONCJ 155.

$R v$ Hutchinson, 2014 NSSC 155.

$R v J A, 2011 \mathrm{SCC} 28$.

$R v J A$, 2011 SCC 28 (Factum of Intervener: Women's Legal Education and Action Fund).

$R v$ Jobidon [1991] 2 SCR 714.

$R v$ Leclerc [1991] OJ No 1533 (Ont CA).

$R v$ Mabior, 2012 SCC 47.

$R v$ Park [1995] 2 SCR 836.

$R v$ Sansregret [1985] 1 SCR 570. 
$R v$ Ururyar, 2016 ONCJ 448.

$R v$ Welch [1995] 25 OR (3d) 665 (CA).

$\underline{\text { Scholarly Sources }}$

Abdullah-Khan, Noreen. Male Rape: The Emergence of a Social and Legal Issue. New York, NY: Palgrave MacMillan, 2008.

Altheide, David. Qualitative Media Analysis. Thousand Oaks, CA: Sage Publications, 1996.

Altheide, David, and Christopher Schneider. Qualitative Media Analysis. 2nd ed. Thousand Oaks, CA: Sage Publications, 2013.

Asimow, Michael. "Popular Culture and the Adversary System." Loyola of Los Angeles Law Review 40, no. 2 (2006): 653- 86.

Bakhtin, Mikhail. Rabelais and His World. Translated by Helene Iswolsky. Bloomington, IN: Indiana University Press, 1984.

Bell, David, and Jon Binnie. The Sexual Citizen: Queer Theory and Beyond. Cambridge, MA: Polity Press, 2000.

Benedet, Janine. "Law Professor Janine Benedet Talks about Ghomeshi." Interview. Video file, 04:21. CTVNews.ca. Posted November 27, 2014. https://bc.ctvnews.ca/video?clipId=499854.

__. "Little Sisters Book and Art Emporium v. Minister of Justice: Sex Equality and the Attack on R. v. Butler." Osgoode Hall Law Journal 39 (2001): 187-205.

Beres, Melanie. "'Spontaneous' Sexual Consent: An Analysis of Consent Literature." Feminism and Psychology 17, no. 1 (2007).

Bergstrom, Kelly. "'Don't Feed the Troll”: Shutting down Debate about Community Expectations on Reddit.com." First Monday 16, no. 8 (2011).

Best, Joel. "The Problems with Moral Panic: The Concept's Limitations." In The Ashgate Research Companion to Moral Panics, edited by Charles Krinsky. London: Routledge, 2016.

Bourdieu, Pierre. Language and Symbolic Power. Cambridge, MA: Polity Press, 1991. 
Bourke, Joanna. Rape: Sex, Violence, History. Emeryville, CA: Shoemaker \& Hoard, 2007.

Bradley, Elizabeth, Leslie Curry, and Kelly Devers. "Qualitative Data Analysis for Health Services Research: Developing Taxonomy, Themes, and Theory." Health Services Research 42, no. 4 (January 24, 2007): 1758-72.

Brownmiller, Susan. Against Our Will: Men, Women, and Rape. New York, NY: Simon and Schuster, 1975.

Busby, Karen. "Every Breath You Take: Erotic Asphyxiation, Vengeful Wives, and Other Enduring Myths in Spousal Sexual Assault Prosecutions." Canadian Journal of Women and the Law 24, no. 2 (2012): 328-58.

The Canadian Press. "Victoria Event Space Hires 'Consent Captain' to Help Prevent Sexual Assaults." CP24. Last modified May 8, 2018. https://www.cp24.com/news/victoria-event-space-hires-consent-captain-to-helpprevent-sexual-assaults-1.3919807.

Cohen, Stanley. Folk Devils and Moral Panics. 1972. Reprint, London: Routledge, 2011.

Cole, Susan. Pornography and the Sex Crises. Toronto: Amanita Press, 1989.

Confino, Alon. "Collective Memory and Cultural History: Problems of Method." The American Historical Review 102, no. 5 (1997): 1386-403.

Cossman, Brenda. Sexual Citizens: The Legal and Cultural Regulation of Sex and Belonging. Stanford, CA: Stanford University Press, 2007.

Cowling, Mark, and Paul Reynolds, eds. Making Sense of Sexual Consent. New York, NY: Routledge, 2016. First published 2004 by Ashgate Publishing.

Craig, Elaine. "Capacity to Consent to Sexual Risk." New Criminal Law Review 17, no. 1 (2014): 103-34.

Dean, Mitchell. Governmentality: Power and Rule in Modern Society. London, United Kingdom: Sage Publications, 1999.

DiManno, Rosie. "Judge's Ranting Rape Verdict Shows He's behind the Curve." The Star (Toronto), September 15, 2016.

Donath, Judith. "Identity and Deception in the Virtual Community." In Communities in Cyberspace, edited by Mark Smith and Peter Kollock. London, United Kingdom: Routledge, 1999. 
Dunn, Caroline. "Damsels in Distress or Partners in Crime?: The Abduction of Women in Medieval England." PhD diss., Fordham University, 2007.

Du Toit, Louise. A Philosophical Investigation of Rape: The Making and Unmaking of the Feminine Self. London: Routledge, 2009.

Dworkin, Andrea. "Against the Male Flood: Censorship, Pornography, and Equality." In Pornography: Private Right or Public Menace?, edited by Robert Baird and Stuart Rosenbaum. New York, NY: Prometheus Books, 1998.

Ehrlich, Susan. "The Discursive Reconstruction of Sexual Consent." Discourse and Society 9, no. 2 (1998): 149-71.

Elo, Satu, and Helvi Kyngäs. "The Qualitative Content Analysis Process." Journal of Advanced Nursing 62, no. 1 (April 2008): 109-12.

End Rape on Campus. http://endrapeoncampus.org.

Estrich, Susan. Real Rape. Cambridge, MA: Harvard University Press, 1987.

Fereday, Jennifer, and Eimear Muir-Cochrane. "Demonstrating Rigor Using Thematic Analysis: A Hybrid Approach of Inductive and Deductive Coding and Theme Development." International Journal of Qualitative Methods 5, no. 1 (March 2006): 80-92.

Fortun, Kim. "Foreword to the 25th Anniversary Edition." Foreword to Writing Culture: The Poetics and Politics of Ethnography, edited by James Clifford and George Marcus. 25th ed. 1986. Reprint, University of California Press, 2010.

Galton, Francis. "The Ballot-Box." Nature 75 (March 1907): 509-10.

Gilbert, Eric. "Widespread Underprovision on Reddit." CSCW'13: Proceedings of the 2013 Conference on Computer Supported Cooperative Work, 2013, 803-08. doi:10.1145/2441776.2441866.

Goode, Erich, and Nachman Ben-Yehuda. "The Genealogy and Trajectory of the Moral Panic Concept." In The Ashgate Research Companion to Moral Panics, edited by Charles Krinsky. London: Routledge, 2016.

- Moral Panics: The Social Construction of Deviance. Oxford: Blackwell, 1994.

Gotell, Lise. "Governing Heterosexuality through Specific Consent: Interrogating the Governmental Effects of R. v. J.A." Canadian Journal of Women and the Law 24, no. 2 (2012a): 359-88. 
. "Rethinking Affirmative Consent in Canadian Sexual Assault Law: Neoliberal Sexual Subjects and Risky Women." Akron Law Review 41, no. 4 (January 2008): 865-98.

. "Third-Wave Anti-rape Activism on Neoliberal Terrain: The Garneau Sisterhood." In Sexual Assault in Canada: Law, Legal Practice and Women's Activism, edited by Elizabeth Sheehy, 243-65. Ottawa: University of Ottawa Press, 2012b.

Grinberg, Emanuella. "The issue with ousted CBC host Jian Ghomeshi's 'kinky defense."' CNN. Last modified November 2, 2014. Accessed May 4, 2018. https://www.cnn.com/2014/10/28/living/jian-ghomeshi-cbc-firedbdsm/index.html.

Gunn, Rita, and Candice Minch. Sexual Assault: The Dilemma of Disclosure, the Question of Conviction. Winnipeg: University of Manitoba Press, 1988.

Gunsaullus, Jennifer. Jennifer Gunsaullus to LinkedIn web forum, "Sexual Consent, Female Sexuality as a Commodity, and Jaclyn Friedman," November 24, 2015. Accessed May 5, 2018. https:/www.linkedin.com/pulse/sexual-consent-femalesexuality-commodity-jaclyn-gunsaullus-ph-d-.

Haggerty, Kevin. "From Risk to Precaution: The Rationalities of Personal Crime Prevention." In Risk and Morality, edited by Richard Ericson and Aaron Doyle, 193-215. Toronto: University of Toronto Press, 2003.

Halberstam, Judith. In a Queer Time and Place. New York, NY: New York University Press, 2005.

Halley, Janet. Split Decisions: How and Why to Take a Break from Feminism. Princeton, NJ: Princeton University Press, 2006.

Hammersley, Martyn, and Paul Atkinson. Ethnography: Principles in Practice. New York, NY: Tavistock Publications, 1983.

Hartman, Yvonne. "In Bed with the Enemy: Some Ideas on the Connections between Neoliberalism and the Welfare State." Current Sociology 53, no. 1 (2005): 57-73.

Hegel, Georg Wilhelm Friedrich. Hegel: Elements of the Philosophy of Right. Cambridge, MA: Cambridge University Press, 1991.

Hilberman, Elaine. The Rape Victim. Washington, DC: American Psychiatric Association, 1976.

Hunt, Alan. Governing Morals: A Social History of Moral Regulation. Cambridge, MA: Cambridge University Press, 1999. 
James, E. L. Fifty Shades Darker. London: Random House, 2012a.

—. Fifty Shades Freed. London: Random House, 2012b.

- Fifty Shades of Grey. London: Random House, 2012c.

Javaid, Aliraza. "Feminism, Masculinity and Male Rape: Bringing Male Rape 'Out of the Closet'." Journal of Gender Studies 25, no. 3 (August 2014): 283-93.

_. "Police Responses To, and Attitudes Towards, Male Rape: Issues and Concerns." International Journal of Police Science and Management 17, no. 2 (2015): 81-90.

_. "Voluntary Agencies' Responses To, and Attitudes toward Male Rape: Issues and Concerns." Sexuality and Culture 20, no. 3 (2016): 731-48.

Jochelson, Richard, James Gacek, and Lauren Menzie. Criminal Law and Precrime: Legal Studies in Canadian Punishment and Surveillance in Anticipation of Criminal Guilt. New York, NY: Routledge, 2018.

Jochelson, Richard, and Kirsten Kramar. Sex and the Supreme Court. Winnipeg: Fernwood, 2011.

Kamir, Orit. Framed: Women in Law and Film. Durham, NC: Duke University Press, 2006.

Khan, Ummni. "Grappling with Ghomeshi." University of Toronto Press Blog. Entry posted November 11, 2014a.

—. "Hot for Kink, Bothered by the Law: BDSM and the Right to Autonomy." The Canadian Bar Association: Alberta Branch (blog). Entry posted August 8, $2016 \mathrm{a}$. Accessed May 5, 2018. https://www.cba-alberta.org/PublicationsResources/Resources/Law-Matters/Law-Matters-Summer-2016-Issue/Hot-forKink,-Bothered-by-the-Law-BDSM-and-the-Rig.

—. "The Rhetoric of Rape Culture." Centre for Free Expression (blog). Entry posted December 5, 2016b. https://cfe.ryerson.ca/blog/2016/12/rhetoric-rape-culture.

— . "Slipping between Danger, Pleasure and the Law: Thoughts on Three Recent Books Addressing Sexuality." Review of a book. Dalhousie Law Journal 37, no. 1 (Spring 2014b): 421-30.

—. "Take My Breath Away: Competing Contexts between Domestic Violence, Kink and the Criminal Justice System in R. v. J.A." Oñati Socio-legal Series 6, no. 6 (2016c): 1405-25. 
- Vicarious Kinks: S/M in the Socio-Legal Imaginary. Buffalo, NY: University of Toronto Press, 2014c.

Kling, Rob, Ya-Ching Lee, Al Teich, and Mark Frankel. "Assessing Anonymous Communication on the Internet: Policy Deliberations." Information Society 15, no. 2 (1999): 79-90.

Knight, Demi. "Lethbridge School District Supports the Early Introduction of Consent into Curricula." Global News. Last modified May 2, 2018.

https://globalnews.ca/news/4182924/lethbridge-school-districts-alberta-consentcurriculum/.

Koshan, Jennifer. "Marriage and Advance Consent to Sex: A Feminist Judgment in R v JA." Oñati Socio-legal Series 6, no. 6 (2016): 1377-404.

Los, M. "The Struggle to Redefine Rape in the Early 1980s." In Confronting Sexual Assault: A Decade of Legal and Social Change, edited by Julian V. Roberts and Renate M. Mohr, 20-56. Toronto: University of Toronto Press, 1994.

Machura, Stefan, and Stefan Ulbrich. "Law in Film: Globalizing the Hollywood Courtroom Drama." In Law and Film, edited by Stefan Machura and Peter Robson. Bristol, United Kingdom: Blackwell Publishers, 2001.

MacIntyre, Alasdair. "The Virtues, the Unity of Human Life, and the Concept of a Tradition." In Liberalism and Its Critics, edited by Michael J. Sandel, 125-48. New York, NY: New York University Press, 1984.

MacKinnon, Catharine. Feminism Unmodified. Cambridge, MA: Harvard University Press, 1987.

—_. "Sexuality, Pornography, and Method: 'Pleasure under Patriarchy."' Ethics 99, no. 2 (January 1989): 314-46.

Mahoney, K. "Canaries in a Coalmine: Canadian Judges and the Reconstruction of Obscenity Law." In Freedom of Expression and the Charter, edited by David Schneiderman. Toronto: Thompson, 1991.

Marwick, Alice, and Danah Boyd. "I Tweet Honestly, I Tweet Passionately: Twitter Users, Context Collapse, and the Imagined Audience." New Media and Society 13 (2011): 114-33. doi:10.1177/1461444810365313.

Marx, Gary. "What's in a Name? Some Reflections on the Sociology of Anonymity." Information Society 15, no. 2 (1999): 99-112. 
Massanari, Adrienne. "\#Gamergate and The Fappening: How Reddit's Algorithm, Governance, and Culture Support Toxic Technocultures." New Media and Society 19, no. 3 (2017): 329-46.

- Participatory Culture, Community, and Play: Learning from Reddit. New York, NY: Peter Lang, 2015.

_. "Playful Participatory Culture: Learning from Reddit." Selected Papers of Internet Research 14 (2013): 1-7.

Matlock, Nicole A. K. "Reasonable Rage: The Problem with Stereotypes in Provocation Cases." Washington University Jurisprudence Review 6, no. 2 (2014): 371-96.

McClung Workman, Hallie. "Formation of Safe Spaces in Gendered Online Communities: Reddit and the "Front Page of the Internet"." PhD diss., Texas Christian University, 2014.

Menzie, Lauren. "Conscious Representations: Critically Interrogating the Engagements and Representations of Extralegal Discourse in R. v. J.A." The Annual Review of Interdisciplinary Justice Research 7 (2018): 292-314.

Mill, John Stuart. On Liberty. 1859. Reprint, Kitchener: Batoche Books, 2001.

Miller, Toby. "Tracking Moral Panic as a Concept." In The Ashgate Research Companion to Moral Panics, edited by Charles Krinsky. London: Routledge, 2016.

Muehlenhard, Charlene L., and Zoe D. Peterson. "III. Wanting and Not Wanting Sex: The Missing Discourse of Ambivalence." Feminism and Psychology 15, no. 1 (2005): 15-20.

Nissenbaum, Helen. "The Meaning of Anonymity in an Information Age." The Information Society 15 (1999): 141-44. doi:10.1080/019722499128592.

O'Connor, Julia, Ann Orloff, and Sheila Shaver. States, Markets, Families: Gender, Liberalism and Social Policy in Australia, Canada, Great Britain and the United States. Cambridge, MA: Cambridge University Press, 1999.

O'Malley, Pat. Risk, Uncertainty and Government. London, United Kingdom: Glass House Press, 2004.

Patel, Arti. "Parents Should Ask Babies for Consent before Diaper Changes, Expert Says - Here's What She Meant." Global News. Last modified May 11, 2018. https://globalnews.ca/news/4202437/consent-changing-diapers/. 
Petter, Olivia. "Why Consent Apps Don't Work, According to Criminal Lawyers." Independent. Last modified May 14, 2018. https://www.independent.co.uk/lifestyle/consent-apps-problems-dating-uconsent-sexual-assault-legal-courta8332706.html.

Pfeuffer, Charyn. "Fifty Shades versus BDSM: The reality of consent." The Globe and Mail. Last modified February 2, 2017. Accessed May 5, 2018. https://www.theglobeandmail.com/life/relationships/valentines-day/fifty-shadesversus-bdsm-the-reality-of-consent/article33876672/.

Phelan, Shane. Sexual Strangers: Gays, Lesbians, and Dilemmas of Citizenship. Philadelphia, PA: Temple University Press, 2001.

Plaxton, Michael. Implied Consent and Sexual Assault: Intimate Relationships, Autonomy, and Voice. Montreal: McGill-Queen's University Press, 2015.

Plummer, Ken. Intimate Citizenship: Private Decisions and Public Dialogues. Seattle, WA: University of Washington Press, 2003.

Richardson, Diane. "Sexuality and Citizenship." Sociology 32, no. 1 (February 1, 1998): 83-100. doi:10.1177/0038038598032001006.

Rimmon-Kenan, Shlomit. Narrative Fiction: Contemporary Poetics. New York, NY: Methuen, 1983.

Roiphe, Katie. The Morning After: Sex, Fear, and Feminism. Boston, NY: Back Bay Books, 1994.

Rose, Nikolas. Governing the Soul: The Shaping of the Private Self. New York, NY: Free Association Books, 1999.

. "Government and Control." The British Journal of Criminology 40, no. 2 (March 2000): 321-39.

Rose, Nikolas, Pat O'Malley, and Mariana Valverde. "Governmentality." Annual Review of Law and Social Science 2 (2006): 83-104.

Salter, Michael, and Thomas Crofts. "Responding to Revenge Porn: Challenging Online Legal Impunity." In New Views on Pornography: Sexuality, Politics and the Law, edited by L. Comella and S. Tarrant, 1-18. Westport: Praeger Publisher, 2015, forthcoming.

Sarah, Emily, Ronald H L Elliston, and Jon Blue. "Fifty Shades of Grey: what BDSM enthusiasts think." Interview by Anna Smith. The Guardian. Last modified February 15, 2015. https://www.theguardian.com/film/2015/feb/15/fifty-shadesof-grey-bdsm-enthusiasts. 
Sheehy, Elizabeth, and Daphne Gilbert. "Responding to Sexual Assault on Campus: What Can Canadian Universities Learn from US Law and Policy?" Social Science Research Network, 2015.

Simon, William, and John Gagnon. "Sexual Scripts: Origins, Influences and Changes." Qualitative Sociology 26, no. 4 (December 2003): 491-97.

_. "Sexual Scripts: Permanence and Change." Archives of Sexual Behavior 15, no. 2 (1986): 97-120.

Singer, Philipp, Fabian Flöck, Clemens Meinhart, Elias Zeitfogel, and Markus Strohmaier. "Evolution of Reddit: From the Front Page of the Internet to a SelfReferential Community?" $W W W$ '14 Companion: Proceedings of the 23rd International Conference on World Wide Web, April 2014, 517-22. doi:10.1145/2567948.2576943.

Stemple, Lara, Andrew Flores, and Ilan H. Meyer. "Sexual Victimization Perpetrated by Women: Federal Data Reveal Surprising Prevalence." Aggression and Violent Behavior 34 (2017): 302-11.

Stuart, Don. "Sexual Assault: Substantive Issues before and after Bill C-49." Criminal Law Quarterly 35 (1992): 241-62.

Stychin, Carl. Governing Sexuality: The Changing Politics of Citizenship and Law Reform. Oxford and Portland, OR: Hart Publishing, 2003.

Surowiecki, James. The Wisdom of Crowds. New York, NY: Anchor Books, 2005. First published 2004 by Random House Inc.

Tang, Kwong-leung. "Rape Law Reform in Canada: The Success and Limits of Legislation." International Journal of Offender Therapy and Comparative Criminology 42, no. 3 (September 1, 1998): 258-70. doi: $10.1177 / 0306624 X 9804200307$.

Tirosh, Noam. "Reconsidering the 'Right to Be Forgotten' - Memory Rights and the Right to Memory in the New Media Era." Media, Culture and Society 39, no. 5 (2017): 644-60.

Turner, Bryan. "Postmodern Culture/Modern Citizen." In The Condition of Citizenship, by Bart Van Steenbergen, 153-68. London, United Kingdom: Sage Publications, 1994.

Ubelacker, Sheryl. "BDSM is about consent and communication - not abuse, say adherents." Global News. Last modified October 30, 2014. 
https://globalnews.ca/news/1644406/bdsm-is-about-consent-and-communicationnot-abuse-say-adherents/.

van der Nagel, Emily. "Faceless Bodies: Negotiating Technological and Cultural Codes on reddit gonewild." Journal of Media Arts Culture 10, no. 2 (2013).

Weninger, Tim, Thomas James Johnston, and Maria Glenski. "Random Voting Effects in Social-Digital Spaces: A Case Study of Reddit Post Submissions." HT '15:

Proceedings of the 26th ACM Conference on Hypertext and Social Media, 2015.

Young, Jock. The Drugtakers: The Social Meaning of Drug Use. London: MacGibbon and Kee, 1971a.

- "Moral Panic: Its Origins in Resistance, Ressentiment, and the Translation of Fantasy into Reality." British Journal of Criminology 49, no. 1 (2009): 4-16.

—. "The Role of the Police as Amplifiers of Deviancy, Negotiators of Reality and Translators of Fantasy." In Images of Deviance, edited by Stanley Cohen. London: Penguin Books, 1971b.

Zanin, Andrea. "A Kinkster's Take on the Jian Ghomeshi Saga." The Tyee. Last modified October 28, 2014. https://thetyee.ca/Opinion/2014/10/28/Kinkster-onJian-Ghomeshi/.

Zuckerman, Esther. "'Why Is Reddit So Anti-Women?': An Epic Reddit Thread Counts the Ways." The Atlantic. Last modified July 26, 2012. https://www.theatlantic.com/entertainment/archive/2012/07/why-reddit-so-antiwomen-epic-reddit-thread-counts-ways/325357/. 


\section{Appendices}

\section{Appendix A}

\section{Codebook}

Commentary on Space (virtual)

Educating, Consent Frameworks

External Solution Proposed

$>$ Cops, Reporting

$>$ Legal Remedy

$>$ Penology, Prison

$>$ Therapy

$>$ Violence

Feminism, Rape Culture

Legal v. Social Understandings

Legal Standards

$>$ Advanced Consent

$>$ Implied Consent

$>$ Jurisdiction

$>$ Terminology

Moral Standards

OP Comments

$>$ Additional Questions

$>$ Arguing

$>$ Clarification

$>$ Judgement Rendered

Questioning purpose of virtual trial

Redditor Input

$>$ Affirming, Yes

$>$ Denying, No

$>$ Unclear, Grey Area

Redditor Self-Identification

$>$ Assaulted

$>$ Assaulter

$>$ Lawyer

$>$ Not a Lawyer

$>$ Service Provider, Counselor

$>$ Survivor

$>$ Victim

Trolling

Victim Blaming 